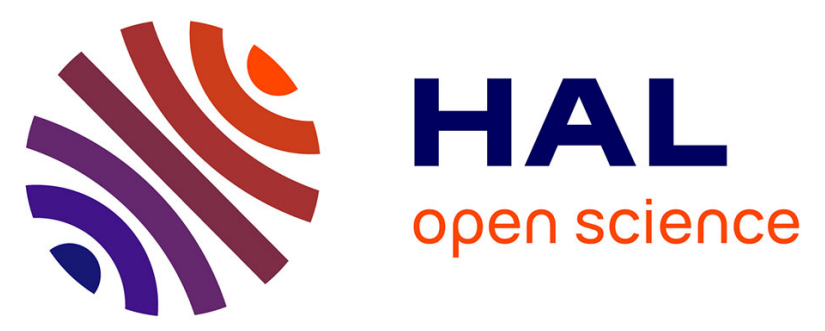

\title{
On the overall behavior, microstructure evolution, and macroscopic stability in reinforced rubbers at large deformations: II-Application to cylindrical fibers
}

Oscar Lopez-Pamies, Pedro Ponte Castañeda

\section{- To cite this version:}

Oscar Lopez-Pamies, Pedro Ponte Castañeda. On the overall behavior, microstructure evolution, and macroscopic stability in reinforced rubbers at large deformations: II-Application to cylindrical fibers. Journal of the Mechanics and Physics of Solids, 2006, 54, pp.831-863. 10.1016/j.jmps.2005.10.010 . hal-00111467

\author{
HAL Id: hal-00111467 \\ https://hal.science/hal-00111467
}

Submitted on 24 Jul 2019

HAL is a multi-disciplinary open access archive for the deposit and dissemination of scientific research documents, whether they are published or not. The documents may come from teaching and research institutions in France or abroad, or from public or private research centers.
L'archive ouverte pluridisciplinaire HAL, est destinée au dépôt et à la diffusion de documents scientifiques de niveau recherche, publiés ou non, émanant des établissements d'enseignement et de recherche français ou étrangers, des laboratoires publics ou privés. 


\title{
On the overall behavior, microstructure evolution, and macroscopic stability in reinforced rubbers at large deformations: II-Application to cylindrical fibers
}

\author{
O. Lopez-Pamies ${ }^{\mathrm{a}, \mathrm{b}}$, P. Ponte Castañeda $\mathrm{a}^{\mathrm{a}, \mathrm{b}, *}$ \\ a Department of Mechanical Engineering and Applied Mechanics, University of Pennsylvania, Philadelphia, \\ PA 19104-6315, USA \\ ${ }^{\mathrm{b}}$ LMS (CNRS UMR 7649), Département de Mécanique, École Polytechnique, 91128 Palaiseau, France
}

\begin{abstract}
In Part I of this paper, we presented a general homogenization framework for determining the overall behavior, the evolution of the underlying microstructure, and the possible onset of macroscopic instabilities in fiber-reinforced elastomers subjected to finite deformations. In this work, we make use of this framework to generate specific results for general plane-strain loading of elastomers reinforced with aligned, cylindrical fibers. For the special case of rigid fibers and incompressible behavior for the matrix phase, closed-form, analytical results are obtained. The results suggest that the evolution of the microstructure has a dramatic effect on the effective response of the composite. Furthermore, in spite of the fact that both the matrix and the fibers are assumed to be strongly elliptic, the homogenized behavior is found to lose strong ellipticity at sufficiently large deformations, corresponding to the possible development of macroscopic instabilities [Geymonat, G., Mü ller, S., Triantafyllidis, N., 1993. Homogenization of nonlinearly elastic materials, macroscopic bifurcation and macroscopic loss of rank-one convexity. Arch. Rat. Mech. Anal. 122, 231-290]. The connection between the evolution of the microstructure and these macroscopic instabilities is put into evidence. In particular, when the reinforced elastomers are loaded in
\end{abstract}

\footnotetext{
${ }^{*}$ Corresponding author. Department of Mechanical Engineering and Applied Mechanics, University of Pennsylvania, Philadelphia, PA 19104-6315, USA.

E-mail address: ponte@seas.upenn.edu (P. Ponte Castañeda).
} 
compression along the long, in-plane axis of the fibers, a certain type of "flopping" instability is detected, corresponding to the composite becoming infinitesimally soft to rotation of the fibers.

Keywords: Microstructures; Finite strain; Rubber material; Particulate reinforced material; Stability and bifurcation

\section{Introduction}

In the preceding paper (Lopez-Pamies and Ponte Castañeda, 2006), henceforth referred to as Part I, we established a homogenization-based framework for determining the overall behavior, the evolution of the underlying microstructure, and the possible onset of macroscopic instabilities in fiber-reinforced elastomers subjected to finite deformations. In particular, general expressions were provided for the case of isotropic elastomers reinforced with compliant or rigid fibers.

The aim of this work is to make use of the framework developed in Part I for the first time. In this light, the selected application in this work corresponds to general, plane-strain loading of (in)compressible elastomers reinforced with aligned, cylindrical fibers of initially elliptical cross section. This application will be shown to be general enough to contain all the essential features of the problem, including the subtle interplay between the evolution of the underlying microstructure and the effective behavior, which can lead to the possible development of macroscopic instabilities, at sufficiently large deformation. At the same time, this application is simple enough that it will allow for a more transparent mathematical analysis - to the extent that it will lead to analytical solutions.

In the next section, for convenience and clarity, we summarize the basic elements of the general theory developed in Part I. Sections 3 and 4 deal with the specific application to fiber-reinforced elastomers. The main results of the paper include closed-form, analytical expressions for the homogenized stored-energy function of an incompressible rubber reinforced by aligned, cylindrical, rigid fibers of elliptical cross section (see expressions (27) and (28)), as well as corresponding expressions for the in-plane rotation of the fibers (see expressions (29) and (30)), under general plane-strain conditions. Finally, some general conclusions will be drawn in Section 5.

\section{Second-order estimates for fiber-reinforced elastomers}

In Part I of this paper, estimates were derived for the effective stored-energy function $\widehat{W}$ of two-phase elastomeric composites consisting of compliant ellipsoidal particles, with given initial volume fraction $c_{0}^{(2)}=c_{0}$ and characterized by the isotropic stored-energy function $W^{(2)}$, which are initially distributed with "ellipsoidal symmetry" (Willis, 1977) in a compressible elastomeric matrix with isotropic stored-energy function $W^{(1)}$. In this section, we summarize briefly the results from Part I that are essential to our purposes. 


\subsection{Compliant fibers}

The second-order estimate for the effective stored-energy function $\widehat{W}$ of the above class of particle-reinforced elastomers is given by

$$
\widehat{W}(\overline{\mathbf{F}})=\left(1-c_{0}\right)\left[W^{(1)}\left(\hat{\mathbf{F}}^{(1)}\right)-\mathscr{S}^{(1)}(\overline{\mathbf{F}}) \cdot\left(\hat{\mathbf{F}}^{(1)}-\overline{\mathbf{F}}^{(1)}\right)\right]+c_{0} W^{(2)}\left(\overline{\mathbf{F}}^{(2)}\right),
$$

where $\mathscr{S}^{(1)}=\partial W^{(1)} / \partial \mathbf{F}$, and the variables $\overline{\mathbf{F}}^{(1)}, \overline{\mathbf{F}}^{(2)}$, and $\hat{\mathbf{F}}^{(1)}$ are functions of the applied macroscopic deformation $\overline{\mathbf{F}}$, of the material properties and of the initial microstructure. They are computed, in a manner to be made more precise further below, from the average deformation gradients $\langle\mathbf{F}\rangle^{(r)}$ and covariance tensors $\mathbf{C}_{\mathbf{F}}^{(r)}=\left\langle\left(\mathbf{F}-\overline{\mathbf{F}}^{(r)}\right) \otimes\left(\mathbf{F}-\overline{\mathbf{F}}^{(r)}\right)\right\rangle^{(r)}$ $(r=1,2)$ in the phases of a linear comparison composite (LCC) with the same microstructure as the original hyperelastic composite in its undeformed configuration, and with local stored-energy function given by

$$
W_{T}^{(r)}(\mathbf{F})=W^{(r)}\left(\mathbf{F}^{(r)}\right)+\mathscr{S}^{(r)}\left(\mathbf{F}^{(r)}\right) \cdot\left(\mathbf{F}-\mathbf{F}^{(r)}\right)+\frac{1}{2}\left(\mathbf{F}-\mathbf{F}^{(r)}\right) \cdot \mathbf{L}^{(r)}\left(\mathbf{F}-\mathbf{F}^{(r)}\right),
$$

where the variables $\mathbf{F}^{(r)}$ and $\mathbf{L}^{(r)}$ denote certain "reference" deformation gradients and modulus tensors to be defined below.

As discussed in Part I, the effective stored-energy function $\widehat{W}_{T}$ of the relevant two-phase LCC may be given in terms of the effective modulus tensor $\widetilde{\mathbf{L}}$ of the two-phase, linearelastic comparison composite with moduli $\mathbf{L}^{(1)}$ and $\mathbf{L}^{(2)}$, and the same microstructure, in its undeformed configuration, as the hyperelastic composite. In this work, use is made of the Willis-type estimate for the effective modulus $\widetilde{\mathbf{L}}$ (Willis, 1977):

$$
\widetilde{\mathbf{L}}=\mathbf{L}^{(1)}+c_{0}\left[\left(1-c_{0}\right) \mathbf{P}^{(1)}-\left(\mathbf{L}^{(1)}-\mathbf{L}^{(2)}\right)^{-1}\right]^{-1},
$$

where $\mathbf{P}^{(1)}$ is a microstructural tensor, defined in Part $\mathbf{I}$, depending on $\mathbf{L}^{(1)}$ and on the symmetric, second-order tensor $\mathbf{Z}_{0}$ serving to characterize the "ellipsoidal symmetry" of the microstructure in the reference configuration.

Having characterized the effective behavior of the LCC, the local properties of the phases in the LCC, as defined by the variables $\mathbf{F}^{(r)}$ and $\mathbf{L}^{(r)}$, are then related to the properties of the actual hyperelastic composite. Thus, the reference deformation gradient tensors are prescribed to be $\mathbf{F}^{(1)}=\overline{\mathbf{F}}$ and $\mathbf{F}^{(2)}=\overline{\mathbf{F}}^{(2)}$, where $\overline{\mathbf{F}}^{(2)}$ denotes the average deformation gradient in the inclusion phase of the LCC, as determined implicitly by the relation (see Part I):

$$
\mathbf{D}^{(1)}\left(\overline{\mathbf{F}}-\overline{\mathbf{F}}^{(2)}\right)+\left(1-c_{0}\right)\left[\mathscr{S}^{(1)}(\overline{\mathbf{F}})-\mathscr{S}^{(2)}\left(\overline{\mathbf{F}}^{(2)}\right)\right]=\mathbf{0},
$$

where

$$
\mathbf{D}^{(1)}=\left(\mathbf{P}^{(1)}\right)^{-1}-\left(1-c_{0}\right) \mathbf{L}^{(1)}
$$

is a fourth-order tensor with major symmetry depending only on $\mathbf{L}^{(1)}$ and on the microstructure. Also, $\overline{\mathbf{F}}^{(1)}$ can be determined in terms of $\overline{\mathbf{F}}^{(2)}$ via

$$
\overline{\mathbf{F}}^{(1)}=\frac{1}{1-c_{0}}\left(\overline{\mathbf{F}}-c_{0} \overline{\mathbf{F}}^{(2)}\right) .
$$

On the other hand, the modulus $\mathbf{L}^{(1)}$ of the matrix phase in the LCC is determined by the "generalized secant" condition

$$
\mathscr{S}^{(1)}\left(\hat{\mathbf{F}}^{(1)}\right)-\mathscr{S}^{(1)}(\overline{\mathbf{F}})=\mathbf{L}^{(1)}\left(\hat{\mathbf{F}}^{(1)}-\overline{\mathbf{F}}\right),
$$

while the modulus in the inclusion phase is given by $\mathbf{L}^{(2)}=\partial^{2} W^{(2)}\left(\overline{\mathbf{F}}^{(2)}\right) / \partial \mathbf{F}^{2}$. 
Finally, the variable $\hat{\mathbf{F}}^{(1)}$, also needed in (1), is determined from the relations (see Part I)

$$
\left(\hat{\mathbf{F}}^{(1)}-\overline{\mathbf{F}}\right) \cdot \frac{\partial \mathbf{L}^{(1)}}{\partial \ell_{\alpha}^{*}}\left(\hat{\mathbf{F}}^{(1)}-\overline{\mathbf{F}}\right)=\frac{c_{0}}{\left(1-c_{0}\right)^{2}}\left(\overline{\mathbf{F}}-\overline{\mathbf{F}}^{(2)}\right) \cdot \frac{\partial \mathbf{D}^{(1)}}{\partial \ell_{\alpha}^{*}}\left(\overline{\mathbf{F}}-\overline{\mathbf{F}}^{(2)}\right),
$$

where the variables $\ell_{\alpha}^{*}(\alpha=1,2, \ldots, 9)$ denote the nine independent components of the fourth-order tensor $\mathbf{L}^{*}$, which is defined by

$$
L_{i j k l}^{(1)}=\bar{Q}_{r m} \bar{Q}_{j n} \bar{Q}_{s p} \bar{Q}_{l q} \bar{R}_{i r} \bar{R}_{k s} L_{m n p q}^{*} .
$$

In this relation, $\overline{\mathbf{R}}$ is the rotation tensor in the polar decomposition of $\overline{\mathbf{F}}=\overline{\mathbf{R}} \overline{\mathbf{U}}$, and $\overline{\mathbf{Q}}$ is the proper-orthogonal, second-order tensor describing the orientation of the Lagrangian principal axes (i.e., the principal axes of $\overline{\mathbf{U}}$ ) via the relation $\overline{\mathbf{U}}=\overline{\mathbf{Q}} \overline{\mathbf{D}}^{\mathrm{Q}}{ }^{\mathrm{T}}$. Recall that for an isotropic matrix phase $\mathbf{L}^{*}$ should be taken to be orthotropic with respect to the Lagrangian principal axes, so that the choice of the independent components $\ell_{\alpha}^{*}(\alpha=1,2, \ldots, 9)$ imposes three internal constraints among the 12 standard components of the orthotropic tensor $\mathbf{L}^{*}$, as explained in more detail in the next section in the context of an example.

In summary, conditions (4), (6), (7) and (8), together with (9), provide a consistent set of equations for the variables $\overline{\mathbf{F}}^{(1)}, \overline{\mathbf{F}}^{(2)}, \hat{\mathbf{F}}^{(1)}$, and $\mathbf{L}^{(1)}$, in terms of which the effective storedenergy function (1) may be readily computed.

\subsection{Rigid fibers}

The above expressions simplify considerably when the inclusions are taken to be rigid. In this limiting case, the particles are constrained to undergo an average rigid rotation $\overline{\mathbf{F}}^{(2)}=\overline{\mathbf{R}}^{(2)}$, so that the second-order estimate (1) reduces to

$$
\widehat{W}(\overline{\mathbf{F}})=\left(1-c_{0}\right) W^{(1)}\left(\hat{\mathbf{F}}^{(1)}\right)+\mathscr{S}^{(1)}(\overline{\mathbf{F}}) \cdot\left[\overline{\mathbf{F}}-c_{0} \overline{\mathbf{R}}^{(2)}-\left(1-c_{0}\right) \hat{\mathbf{F}}^{(1)}\right],
$$

where the variables $\overline{\mathbf{R}}^{(2)}, \hat{\mathbf{F}}^{(1)}$, and $\mathbf{L}^{(1)}$ are now determined by the "generalized secant" Eq. (7), the following equation for the rotation $\overline{\mathbf{R}}^{(2)}$ of the particles:

$$
\begin{aligned}
& \left(\overline{\mathbf{R}}^{(2)}\right)^{\mathrm{T}}\left[\mathbf{D}^{(1)}\left(\overline{\mathbf{F}}-\overline{\mathbf{R}}^{(2)}\right)\right]-\left[\mathbf{D}^{(1)}\left(\overline{\mathbf{F}}-\overline{\mathbf{R}}^{(2)}\right)\right]^{\mathrm{T}} \overline{\mathbf{R}}^{(2)} \\
& \quad+\left(1-c_{0}\right)\left[\left(\overline{\mathbf{R}}^{(2)}\right)^{\mathrm{T}} \mathscr{S}^{(1)}(\overline{\mathbf{F}})-\left(\mathscr{S}^{(1)}(\overline{\mathbf{F}})\right)^{\mathrm{T}} \overline{\mathbf{R}}^{(2)}\right]=\mathbf{0},
\end{aligned}
$$

and the fluctuation equations

$$
\left(\hat{\mathbf{F}}^{(1)}-\overline{\mathbf{F}}\right) \cdot \frac{\partial \mathbf{L}^{(1)}}{\partial \ell_{\alpha}^{*}}\left(\hat{\mathbf{F}}^{(1)}-\overline{\mathbf{F}}\right)=\frac{c_{0}}{\left(1-c_{0}\right)^{2}}\left(\overline{\mathbf{F}}-\overline{\mathbf{R}}^{(2)}\right) \cdot \frac{\partial \mathbf{D}^{(1)}}{\partial \ell_{\alpha}^{*}}\left(\overline{\mathbf{F}}-\overline{\mathbf{R}}^{(2)}\right),
$$

where it is recalled that $\mathbf{D}^{(1)}$ is given by relation (5), and that the variables $\ell_{\alpha}^{*}$ are defined by relation (9).

\section{Plane-strain loading of fiber-reinforced elastomers}

The results summarized in the previous section are specific for fiber-reinforced, isotropic elastomers with "ellipsoidal" microstructures. However, the results are general as far as the loading conditions are concerned. The aim of this work is to make use of these results for the first time, and hence additional hypothesis will be imposed in an attempt to generate results that are as explicit as possible. Thus, in this section, we will study the problem of 
plane-strain deformations (in the 1-2 plane) of fiber-reinforced elastomers where the cylindrical fibers, which are perpendicular to the plane of the deformation, are aligned in the 3-axis direction. Moreover, the fibers have an initial volume fraction $c_{0}$ and are taken to have an initially elliptical cross section of aspect ratio $\omega_{0}$, and to be initially distributed with "elliptical symmetry," involving equal aspect ratios and orientations for all the fibers, in the plane of deformation. For simplicity and without loss of generality, $\omega_{0}$ will be taken greater than or equal to one in the development that follows. Note that the applied deformation $\overline{\mathbf{F}}$ here is entirely characterized by the four in-plane components: $\bar{F}_{11}, \bar{F}_{22}$, $\bar{F}_{12}$, and $\bar{F}_{21}$, since the out-of-plane components are known: $\bar{F}_{13}=\bar{F}_{23}=\bar{F}_{31}=\bar{F}_{32}=0$, and $\bar{F}_{33}=1$ (see Fig. 1(a)).

Recalling the section on microstructure evolution from Part I, the relevant microstructural variables, in the context of plane-strain loading for the type of fiberreinforced elastomers considered here, are the current values of the volume fraction, $c$, the average in-plane aspect ratio, $\omega$, and the average in-plane orientation of the fibers, $\phi$. Fig. 1(b) shows an schematic representation of the cross section in the plane of deformation of a typical fiber with the various microstructural variables. In this figure, the shaded ellipse represents the 1-2 cross section of a typical fiber with initial aspect ratio $\omega_{0}=z_{2}^{0} / z_{1}^{0}$ in the reference configuration, with the rectangular Cartesian basis $\left\{\mathbf{e}_{i}\right\}$ denoting its principal directions. The dashed ellipse represents the 1-2 cross section of the same fiber in the deformed configuration. In this connection, the current average aspect ratio of the fiber is denoted by $\omega=z_{2} / z_{1}$ and the corresponding average principal directions are denoted by the rectangular Cartesian basis $\left\{\mathbf{e}_{i}^{\prime}\right\}$, whose orientation relative to $\left\{\mathbf{e}_{i}\right\}$ is determined by the angle $\phi$ (which is measured in the anticlockwise sense). (Note that the initial orientation of the fibers corresponds to $\phi_{0}=0$.) For convenience, the basis $\left\{\mathbf{e}_{i}\right\}$ defining the principal direction of the inclusions in the reference configuration will be identified here with the fixed laboratory frame of reference. In the rest of this discussion, the components of any tensorial quantity will be referred to $\left\{\mathbf{e}_{i}\right\}$.
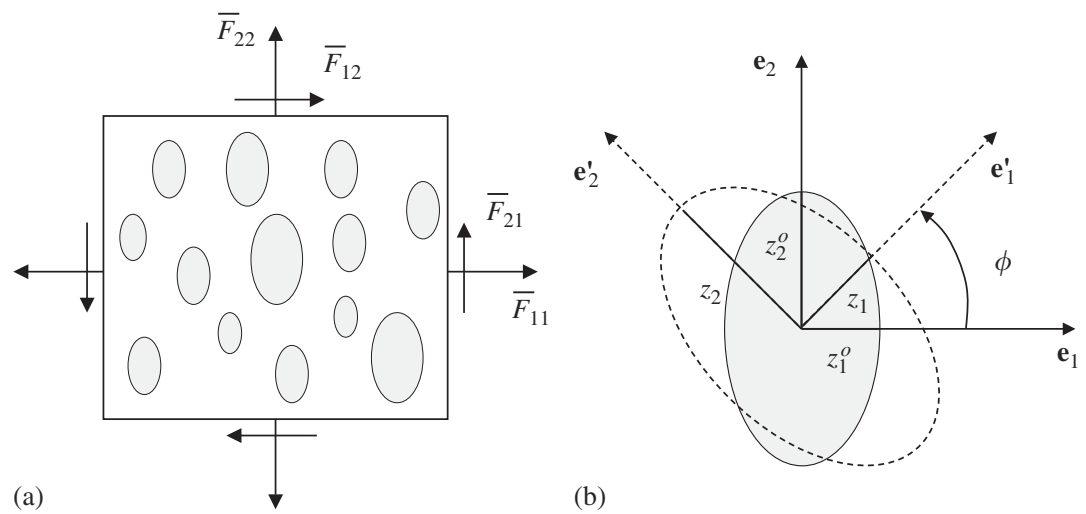

(b)

Fig. 1. Schematic representation of the microstructure of a fiber-reinforced elastomer, depicting the applied loading and the various microstructural variables. (a) The 1-2 cross section of the composite together with the applied loading conditions. (b) The shaded ellipse represents the 1-2 cross section of a typical fiber with initial aspect ratio $\omega_{0}=z_{2}^{0} / z_{1}^{0}$ in the reference configuration; the dashed ellipse corresponds to the $1-2$ cross section of the same fiber in the deformed configuration, with current aspect ratio $\omega=z_{2} / z_{1}$ and current orientation relative to the fixed laboratory frame given by $\phi$. 
Recalling the decompositions $\overline{\mathbf{F}}=\overline{\mathbf{R}} \overline{\mathbf{U}}$ and $\overline{\mathbf{U}}=\overline{\mathbf{Q}} \overline{\mathbf{D}} \overline{\mathbf{Q}}^{\mathrm{T}}$ used in the context of expression (9), it is convenient for later use to introduce angles $\bar{\psi}$ and $\bar{\theta}$, serving to quantify the macroscopic rotation (or "continuum spin") $\overline{\mathbf{R}}$, and the orientation (in the anticlockwise sense relative to the fixed laboratory frame) of the in-plane Lagrangian principal axes (or "loading angle") $\overline{\mathbf{Q}}$, via the expressions

$$
\bar{R}=\left[\begin{array}{cc}
\cos \bar{\psi} & -\sin \bar{\psi} \\
\sin \bar{\psi} & \cos \bar{\psi}
\end{array}\right] \text { and } \bar{Q}=\left[\begin{array}{cc}
\cos \bar{\theta} & -\sin \bar{\theta} \\
\sin \bar{\theta} & \cos \bar{\theta}
\end{array}\right] .
$$

The principal values of $\overline{\mathbf{U}}$ are, of course, the macroscopic principal stretches $\bar{\lambda}_{1}$ and $\bar{\lambda}_{2}$. Thus, the four independent loading parameters $\bar{\psi}, \bar{\theta}, \bar{\lambda}_{1}$ and $\bar{\lambda}_{2}$ are entirely equivalent to the four parameters $\bar{F}_{11}, \bar{F}_{22}, \bar{F}_{12}$, and $\bar{F}_{21}$.

Next, note that the isotropy of the matrix and fiber phases, together with the planestrain conditions, imply that the corresponding stored-energy functions $W^{(r)}(r=1,2)$ may be expressed as functions of the two principal invariants of the right Cauchy-Green deformation tensor $\mathbf{C}=\mathbf{F}^{\mathrm{T}} \mathbf{F}=\mathbf{U}^{2}$ :

$$
I=\operatorname{tr} \mathbf{C}=\lambda_{1}^{2}+\lambda_{2}^{2} \text { and } J=\sqrt{\operatorname{det} \mathbf{C}}=\lambda_{1} \lambda_{2},
$$

or equivalently, as symmetric functions of the associated principal stretches $\lambda_{1}, \lambda_{2}$. Therefore, $W^{(r)}$ may be written as

$$
W^{(r)}(\mathbf{F})=\Psi^{(r)}(I, J)=\Phi^{(r)}\left(\lambda_{1}, \lambda_{2}\right)
$$

However, actual rubbers being nearly incompressible, it will suffice here to consider isotropic phases characterized by stored-energy functions of the type

$$
W^{(r)}(\mathbf{F})=g^{(r)}(I)+h^{(r)}(J)+\frac{\kappa^{(r)}-\mu^{(r)}}{2}(J-1)^{2},
$$

where the parameter $\mu^{(r)}$ denotes the initial shear modulus, and the in-plane bulk modulus $\kappa^{(r)}$ will be taken to tend to infinity in order to recover incompressible behavior $(J \rightarrow 1)$. Here, the material functions $g^{(r)}$ and $h^{(r)}$ are assumed to be twice continuously differentiable and to satisfy the conditions: $g^{(r)}(2)=h^{(r)}(1)=0, g_{I}^{(r)}(2)=\mu^{(r)} / 2, h_{J}^{(r)}(1)=$ $-\mu^{(r)}$, and $4 g_{I I}^{(r)}(2)+h_{J J}^{(r)}(1)=\mu^{(r)}$, where the subscripts $I$ and $J$ denote partial differentiation with respect to these invariants. Under these conditions, $W^{(r)}(\mathbf{F}) \sim\left(\frac{1}{2}\right)\left(\kappa^{(r)}-\mu^{(r)}\right)(\operatorname{tr} \boldsymbol{\varepsilon})^{2}+$ $\mu^{(r)} \operatorname{tr} \boldsymbol{\varepsilon}^{2}$, where $\boldsymbol{\varepsilon}$ is the infinitesimal strain tensor, as $\mathbf{F} \rightarrow \mathbf{I}$, so that the stored-energy function (16) linearizes properly. A relatively simple model of the general type (16), which captures the limiting chain extensibility of elastomers, is the (compressible) Gent model (Gent, 1996):

$$
W(\mathbf{F})=-\frac{\mu J_{m}}{2} \ln \left[1-\frac{I-2}{J_{m}}\right]-\mu \ln J+\left(\frac{\kappa-\mu}{2}-\frac{\mu}{J_{m}}\right)(J-1)^{2},
$$

where the parameter $J_{m}$ is the limiting value for $I-2$ at which the material locks up. Note that (17) reduces to a Neo-Hookean material on taking the limit $J_{m} \rightarrow \infty$. Note also that the stored-energy function (17) is strongly elliptic for plane-strain deformations if $\mu>0$, $J_{m}>0$, and $\kappa>2 \mu / J_{m}+\mu$, which will be assumed here.

Before proceeding with the computation of the second-order estimates, it is useful to report here the formula for the microstructural tensor $\mathbf{P}^{(1)}$, when specialized to cylindrical fibers aligned with the three direction. In this case, the matrix $\mathbf{Z}_{0}$ takes the form 
$Z_{0}=\operatorname{diag}\left(1 / z_{1}^{0}, 1 / z_{2}^{0}, 0\right)$, and the tensor $\mathbf{P}^{(1)}$ reduces to (Willis, 1981):

$$
P_{i j k l}^{(1)}=\frac{\omega_{0}}{2 \pi} \int_{\xi_{1}^{2}+\xi_{2}^{2}=1} \frac{H_{i j k l}^{(1)}\left(\xi_{1}, \xi_{2}, \xi_{3}=0\right)}{\xi_{1}^{2}+\omega_{0}^{2} \xi_{2}^{2}} \mathrm{~d} S,
$$

where $\mathbf{H}^{(1)}$ has been defined in Part I, and $\omega_{0}=z_{2}^{0} / z_{1}^{0}$ characterizes the aspect ratio of the 1-2 cross section of the fibers in the reference configuration. It is emphasized that the components of (18) are relative to the principal axes of the inclusion (in the undeformed configuration). From a computational point of view, $\mathbf{P}^{(1)}$ depends on the anisotropy of the modulus $\mathbf{L}^{(1)}$ (through $\mathbf{H}^{(1)}$ ), which in turn depends on the functional form of the potential $W^{(1)}$, as well as the particular type of loading, as determined by $\overline{\mathbf{F}}$.

\subsection{Second-order homogenization estimates: compliant fibers}

In order to carry out the computation of the second-order estimate (1) for fiberreinforced elastomers under plane-strain deformations, it suffices to restrict the analysis to the in-plane components of a general deformation gradient tensor $\mathbf{F}$. In turn, it suffices to consider the in-plane components of the modulus tensor $\mathbf{L}^{(1)}$ of the matrix phase of the LCC, which may be conveniently expressed as a matrix in $\mathfrak{R}^{4 \times 4}$ :

$$
\left[\begin{array}{llll}
L_{1111} & L_{1122} & L_{1112} & L_{1121} \\
L_{1122} & L_{2222} & L_{2212} & L_{2221} \\
L_{1112} & L_{2212} & L_{1212} & L_{1221} \\
L_{1121} & L_{2221} & L_{1221} & L_{2121}
\end{array}\right],
$$

where, for notational simplicity, the superscript '(1)' has been suppressed for the components of $\mathbf{L}^{(1)}$, and use has been made of the major symmetry (i.e., $L_{i j k l}=L_{k l i j}$ ). Recalling that $\mathbf{L}^{*}$ has been taken to be orthotropic, it follows that it may be written in the form

$$
\left[\begin{array}{cccc}
L_{1111}^{*} & L_{1122}^{*} & 0 & 0 \\
L_{1122}^{*} & L_{2222}^{*} & 0 & 0 \\
0 & 0 & L_{1212}^{*} & L_{1221}^{*} \\
0 & 0 & L_{1221}^{*} & L_{2121}^{*}
\end{array}\right] .
$$

Since $\overline{\mathbf{R}}$ and $\overline{\mathbf{Q}}$ can be readily computed from $\overline{\mathbf{F}}$, prescription (9) entails that $\mathbf{L}^{(1)}$ possesses six independent components, namely, $L_{1111}^{*}, L_{2222}^{*}, L_{1122}^{*}, L_{1212}^{*}, L_{2121}^{*}$, and $L_{1221}^{*}$. For simplicity, guided by the fact that under plane-strain conditions the tensor $\hat{\mathbf{F}}^{(1)}$ has at most four independent components $\left(\hat{F}_{11}^{(1)}, \hat{F}_{22}^{(1)}, \hat{F}_{12}^{(1)}, \hat{F}_{21}^{(1)}\right)$ which should be determined from Eq. (8), it is expedient to reduce the independent components of (20) to just four. Thus, the following constraints are imposed among the components of (20):

$$
L_{2121}^{*}=L_{1212}^{*} \quad \text { and } \quad L_{1221}^{*}=\sqrt{\left(L_{1111}^{*}-L_{1212}^{*}\right)\left(L_{2222}^{*}-L_{1212}^{*}\right)}-L_{1122}^{*} .
$$

As explained in Lopez-Pamies and Ponte Castañeda (2004b), the motivation for the choices (21) is twofold: (i) relations (21) are consistent ${ }^{1}$ with the tangent modulus of a

\footnotetext{
${ }^{1}$ In fact, $(21)_{1}$ is consistent with the tangent modulus tensor of any isotropic hyperelastic material of form (16).
} 
Neo-Hookean material; and (ii) conditions (21) prove useful to simplify the computation of the microstructural tensor $\mathbf{P}^{(1)}$. However, it should be emphasized that, in principle, other choices are possible.

With choice (9) for the modulus $\mathbf{L}^{(1)}$ of the matrix phase of the LCC, conditions (21) for components (20) of $\mathbf{L}^{*}$, and making use of the identifications $\ell_{1}^{*}=L_{1111}^{*}, \ell_{2}^{*}=L_{2222}^{*}$, $\ell_{3}^{*}=L_{1212}^{*}$, and $\ell_{4}^{*}=L_{1122}^{*}$, Eq. (8) can be seen to reduce to four consistent equations for the four components of $\hat{\mathbf{F}}^{(1)}$. These equations are more conveniently expressed in terms of the variable $\mathbf{Y}$ :

$$
\mathbf{Y}=\overline{\mathbf{Q}}^{\mathrm{T}} \overline{\mathbf{R}}^{\mathrm{T}}\left(\hat{\mathbf{F}}^{(1)}-\overline{\mathbf{F}}\right) \overline{\mathbf{Q}},
$$

which leads to the expressions

$$
\begin{aligned}
& \left(Y_{11}\right)^{2}+2 f_{1} Y_{12} Y_{21}=\frac{2}{1-c_{0}} \frac{\partial \widehat{W}_{T}}{\partial L_{1111}^{*}} \doteq k_{1}, \\
& \left(Y_{22}\right)^{2}+2 f_{2} Y_{12} Y_{21}=\frac{2}{1-c_{0}} \frac{\partial \widehat{W}_{T}}{\partial L_{2222}^{*}} \doteq k_{2}, \\
& \left(Y_{12}\right)^{2}+\left(Y_{21}\right)^{2}+2 f_{3} Y_{12} Y_{21}=\frac{2}{1-c_{0}} \frac{\partial \widehat{W}_{T}}{\partial L_{1212}^{*}} \doteq k_{3}, \\
& Y_{11} Y_{22}-Y_{12} Y_{21}=\frac{1}{1-c_{0}} \frac{\partial \widehat{W}_{T}}{\partial L_{1122}^{*}} \doteq k_{4},
\end{aligned}
$$

where $f_{1}, f_{2}, f_{3}, k_{1}, k_{2}, k_{3}, k_{4}$ are functions of the independent components of $\mathbf{L}^{(1)}$, i.e., $L_{1111}^{*}, L_{2222}^{*}, L_{1122}^{*}$, and $L_{1212}^{*}$, as well as of the deformations $\overline{\mathbf{F}}$ and $\overline{\mathbf{F}}^{(2)}$, the initial volume fraction and aspect ratio of the fibers, $c_{0}$ and $\omega_{0}$, and the constitutive functions, $g^{(r)}, h^{(r)}$, and $\kappa^{(r)}$, of the phases. Eqs. (23) can be shown to yield two distinct solutions for $Y_{11}$ and $Y_{22}$, in terms of which $Y_{12}$ and $Y_{21}$ may be determined. Note, however, that the variables $Y_{12}$ and $Y_{21}$ only enter the equations through the combinations $Y_{12} Y_{21}$ and $\left(Y_{12}\right)^{2}+\left(Y_{21}\right)^{2}$, and hence, only these combinations can be determined uniquely from (23). The two solutions for $Y_{11}$ and $Y_{22}$ are as follows:

$$
Y_{11}= \pm \frac{2 f_{1} k_{4}+k_{1}}{\sqrt{4 f_{1}^{2} k_{2}+4 f_{1} k_{4}+k_{1}}}, \quad Y_{22}= \pm \frac{2 f_{1} k_{2}+k_{4}}{\sqrt{4 f_{1}^{2} k_{2}+4 f_{1} k_{4}+k_{1}}},
$$

where it must be emphasized that the positive (and negative) signs must be chosen to go together in the roots for $Y_{11}$ and $Y_{22}$.

Next, using the relation $\hat{\mathbf{F}}^{(1)}=\overline{\mathbf{R}} \overline{\mathbf{Q}} \mathbf{Y} \overline{\mathbf{Q}}^{\mathrm{T}}+\overline{\mathbf{F}}$, each of the two distinct roots for $\mathbf{Y}$ may be substituted into expression (7). The resulting relation, together with expression (4), form a system of eight nonlinear algebraic equations for the eight scalar unknowns $\bar{F}_{11}^{(2)}, \bar{F}_{22}^{(2)}, \bar{F}_{12}^{(2)}, \bar{F}_{21}^{(2)}, L_{1111}^{*}, L_{2222}^{*}, L_{1122}^{*}$, and $L_{1212}^{*}$, which must be solved numerically. It is worth mentioning that by exploiting the objectivity and isotropy of the stored-energy functions of the phases of the composite, the equations obtained from (7) and (4) may be finally cast into a rather simple form. Having computed the values of all the components of $\overline{\mathbf{F}}^{(2)}$ and $\mathbf{L}^{(1)}$ for a given initial fiber concentration $c_{0}$ and aspect ratio $\omega_{0}$, given material 
behavior $g^{(r)}, h^{(r)}$, and $\kappa^{(r)}$, and given loading $\overline{\mathbf{F}}$, the values of the components of $\overline{\mathbf{F}}^{(1)}$ and $\hat{\mathbf{F}}^{(1)}$ can be readily determined using relations (6) and (24), respectively. In turn, these results may be used to compute the second-order estimate (1) for the effective storedenergy function $\widehat{W}$ of the fiber-reinforced elastomers. Also, the evolution of the microstructural variables $c, \omega$, and $\phi$ may be determined from the estimate for $\overline{\mathbf{F}}^{(2)}$, by means of the tensor $\mathbf{Z}=\mathbf{Z}_{0} \overline{\mathbf{F}}^{(2)^{-1}}$, as discussed in Part I. Thus, letting $\mathbf{Z}^{\mathrm{T}} \mathbf{Z}=\mathbf{K} \mathbf{W} \mathbf{K}^{\mathrm{T}}$, where $\mathbf{W}$ is a diagonal tensor with components $z_{1}^{-2}$ and $z_{2}^{-2}$, such that $\omega=z_{2} / z_{1}$, the orthogonal tensor $\mathbf{K}$ defines the fiber-orientation angle $\phi$ (see Fig. 1(b)) via the relation

$$
K=\left[\begin{array}{cc}
\cos \phi & -\sin \phi \\
\sin \phi & \cos \phi
\end{array}\right] .
$$

In connection with these results, it is important to remark that the two above-mentioned roots lead to very similar results for the effective behavior of fiber-reinforced elastomers when both $\kappa^{(r)}(r=1,2)$ and $\omega_{0}$ are finite (of the same order as $\left.\mu^{(1)}\right)$. However, in the limiting case when the microstructure approaches a simple laminate with compressible phases, i.e., for $\omega_{0} \gg 1$ with $\kappa^{(r)}$ and $\mu^{(1)}$ finite, the estimates associated with the "positive" root can be shown to be superior to those associated with the "negative" root, since only they recover the exact effective behavior of simple laminates. On the other hand, for the case when the bulk modulus of the phases is large, and the aspect ratio of the fibers is finite, i.e., for $\kappa^{(r)} \gg 1$ and $\omega_{0}$ finite, it can be shown that only the "negative" root generates physically meaningful estimates. (However, in the incompressible limit, $\kappa^{(r)}=\infty$, both roots recover the exact result for the laminate, $\omega_{0}=\infty$.) Consequently, given that the primary interest here is in rubbers (which are known to be nearly incompressible) reinforced with fibers of finite aspect ratio, the "negative" root should be used in the computation of the second-order estimates for the effective behavior of fiber-reinforced elastomers.

\subsection{Second-order homogenization estimates: rigid fibers}

\subsubsection{Compressible matrix}

The computation of the second-order estimates (10) for the effective behavior of compressible elastomers reinforced with rigid fibers parallels that given in Section 3.1 for the elastomers reinforced by compliant fibers. Indeed, prescribing the same restrictions (21) for the modulus tensor $\mathbf{L}^{(1)}$ of the matrix phase of the LCC, Eq. (12) can be seen to provide four consistent equations for the four components of $\hat{\mathbf{F}}^{(1)}$. These equations have the same form (23) introduced in Section 3.1 in terms of the variable $\mathbf{Y}$, defined by relation (22), where now the corresponding $f_{1}, f_{2}, f_{3}, k_{1}, k_{2}, k_{3}, k_{4}$ are functions of $L_{1111}^{*}, L_{2222}^{*}, L_{1122}^{*}$, $L_{1212}^{*}, \overline{\mathbf{F}}, \overline{\mathbf{R}}^{(2)}$, as well as $c_{0}, \omega_{0}$, and the matrix constitutive functions, $g^{(1)}, h^{(1)}$, and $\kappa^{(1)}$. (Recall that for rigid fibers $\omega=\omega_{0}$.)

Next, each of the two distinct roots of Eq. (23) may be substituted into the generalized secant Eq. (7), using the expression $\hat{\mathbf{F}}^{(1)}=\overline{\mathbf{R}} \overline{\mathbf{Q}} \mathbf{Y} \overline{\mathbf{Q}}^{\mathrm{T}}+\overline{\mathbf{F}}$. The resulting equation, together with expression (11) for the orthogonal tensor $\overline{\mathbf{R}}^{(2)}$ characterizing the average rotation of the fibers $\phi$ (note that $\mathbf{K}=\overline{\mathbf{R}}^{(2)}$ in this case), form a system of five nonlinear algebraic 
equations for the five scalar unknowns $\phi, L_{1111}^{*}, L_{2222}^{*}, L_{1122}^{*}$, and $L_{1212}^{*}$, which must be solved numerically. Having computed the values of these variables, for given fiber concentration $c_{0}$ and aspect ratio $\omega$, given matrix behavior, $g^{(1)}, h^{(1)}$, and $\kappa^{(1)}$, and given loading $\overline{\mathbf{F}}$, the values of the components of $\hat{\mathbf{F}}^{(1)}$ can be readily determined using relation (24). In turn, these results can be used to compute the second-order estimate (10) for the effective stored-energy function $\widehat{W}$ of the rigidly reinforced elastomers. Finally, the same comments apply as in the previous subsection concerning the selection of the roots in expressions (22).

\subsubsection{Incompressible matrix}

The above expressions can be simplified considerably in the limit of incompressible behavior for the matrix phase, i.e., $\kappa^{(1)} \rightarrow \infty$. In this context, it is recalled that the asymptotic behavior of the two above-mentioned "roots" is quite different for large values of $\kappa^{(1)}$. More specifically, in the limit $\kappa^{(1)} \rightarrow \infty$, the second-order estimates associated with the "negative" root can be shown (see Appendix A) to be consistent with the exact overall incompressibility constraint

$$
C(\overline{\mathbf{F}})=\operatorname{det} \overline{\mathbf{F}}-1=0,
$$

whereas the estimates associated with the "positive" root lead to a different constraint, and are therefore inconsistent with the physics of the problem. Having clarified this point, it is noted that under plane-strain conditions the macroscopic incompressibility constraint (26) implies that the principal stretches can be written in the form $\bar{\lambda}_{1}=\bar{\lambda}$ and $\bar{\lambda}_{2}=1 / \bar{\lambda}$, where $\bar{\lambda} \geqslant 1$, so that there is only one loading parameter in this case (apart from the loading angle $\bar{\theta}$ ). It then follows (see Appendix A) that the second-order estimate (10), associated with the "negative" root, for the effective stored-energy function of an incompressible, isotropic elastomer reinforced with aligned, rigid fibers of elliptical cross section, in volume fraction $c=c_{0}$, and with aspect ratio $\omega=\omega_{0},(\omega \geqslant 1)$, reduces to

$$
\widehat{W}^{I}(\overline{\mathbf{F}})=\widehat{W}^{I}(\overline{\mathbf{U}})=\breve{W}(\bar{\lambda}, \bar{\theta})=(1-c) g^{(1)}\left(\hat{I}^{(1)}\right),
$$

where

$$
\begin{aligned}
\hat{I}^{(1)}= & \frac{c\left(1+\bar{\lambda}^{2}\right)^{2}+\left[1+2(c-2) c \bar{\lambda}^{2}+\bar{\lambda}^{4}\right] \omega+c\left(1+\bar{\lambda}^{2}\right)^{2} \omega^{2}}{(1-c)^{2} \bar{\lambda}^{2} \omega} \\
& -\frac{c\left(\bar{\lambda}^{4}-1\right)(\omega-1)}{(1-c)^{2} \bar{\lambda}^{2} \omega} \sin (\varphi) \sin (\varphi-2 \bar{\theta})-\frac{2 c\left(1+\bar{\lambda}^{2}\right)\left(1+\omega^{2}\right)}{(1-c)^{2} \bar{\lambda} \omega} \cos (\varphi) .
\end{aligned}
$$

In this relation, the angle $\varphi$ is given by

$$
\varphi=\phi-\bar{\psi},
$$

and satisfies the kinematical relation

$$
2 \bar{\lambda}\left(1+\omega^{2}\right) \sin (\varphi)-\left(\bar{\lambda}^{2}-1\right)\left(\omega^{2}-1\right) \sin [2(\varphi-\bar{\theta})]=0
$$

Thus, the angle $\varphi$ serves to describe the evolution of the particle orientation $\phi=\varphi+\bar{\psi}$, as a function of the loading parameters $\bar{\lambda}$ and $\bar{\theta}$, for a given value of the fiber aspect ratio $\omega$, via the remarkably simple relation (30).

There are several important remarks that should be made in the context of expression (27) for the effective stored-energy function of the incompressible reinforced elastomer. 
First, this estimate linearizes properly, and therefore recovers the correct linearized moduli of the composite, in agreement with the Willis estimates for incompressible, rigidly reinforced, elastic materials, at small deformations. Second, this estimate can be seen to be consistent with overall objectivity, $\widehat{W}^{I}(\overline{\mathbf{F}})=\widehat{W}^{I}(\overline{\mathbf{U}})$, in view of the dependence on the rotation of the particles through the difference between the "macroscopic" rotation angle $\bar{\psi}$ and the "microstructural" rotation angle $\phi$. This difference is what is known in plasticity as the "plastic spin." Finally, it should be remarked that the stored-energy function (27) has been shown to satisfy the polyconvex, lower bound (Ponte Castañeda, 1989). For conciseness, the corresponding details will be omitted here. The Voigt upper bound (Ogden, 1978) becomes $+\infty$ in this context, so that it is trivially satisfied by (27).

There are also several interesting remarks that may be made in the context of expression (30) for the relevant microstructural variable, $\phi=\bar{\psi}+\varphi$, the average rotation angle of the fibers. (Recall that in this case the volume fraction, $c$, and aspect ratio, $\omega$, of the fibers remain fixed, irrespectively of the applied deformation.) First, it is interesting to remark that the misalignment angle $\varphi$ depends exclusively on the applied strain $(\bar{\lambda}$ and $\bar{\theta})$ and the shape of the fibers $(\omega)$, but not on the constitutive behavior of the matrix phase, nor on the volume fraction of fibers. Second, it can be shown from (30), that $\varphi \rightarrow \bar{\theta}-\pi / 2$ as $\bar{\lambda} \rightarrow \infty$, for all $\bar{\theta} \in(0, \pi)$. That is, as $\bar{\lambda}$ increases, the fibers tend to orient themselves in such a way that their longest in-plane axes tend to become aligned with the tensile loading axis. For the special value of $\bar{\theta}=0$, when the fibers are already (initially) aligned with the loading axes, the fibers do not rotate, but instead, remain fixed in orientation. In particular, this implies that the large-deformation behavior of $\varphi$ has a discontinuity at $\bar{\theta}=0$, corresponding to the situation when the fibers are aligned with the compressive axis, since in this case $\varphi \rightarrow-\pi / 2(\pi / 2)$ as $\bar{\lambda} \rightarrow \infty$, for $\bar{\theta}=0+(0-)$, but $\varphi=0, \forall \bar{\lambda}$, for $\bar{\theta}=0$. In the results section, this behavior will be related to the possible development of symmetrybreaking, macroscopic instabilities, for loading conditions involving compression along the long axes of the fibers.

\subsubsection{Incompressible matrix: special cases}

The second-order estimate (27) is valid for arbitrary fiber cross section. This includes two interesting extreme cases: $\omega \rightarrow \infty$, corresponding to a laminated material, and $\omega=1$, corresponding to an isotropic distribution of circular fibers.

For the laminate case $(\omega \rightarrow \infty)$, the stored-energy function (27) can be shown to become unbounded for all deformations except for simple shear "parallel" to the layers, i.e., $\overline{\mathbf{F}}=\mathbf{I}+\bar{\gamma} \mathbf{e}_{2} \otimes \mathbf{e}_{1}$, where $\bar{\gamma}$ is the amount of macroscopic shear. This is consistent with the fact that this type of deformation is the only one that may be achieved without deforming the rigid phase. It is easy to show that for $\omega \rightarrow \infty$ and $\overline{\mathbf{F}}=\mathbf{I}+\bar{\gamma} \mathbf{e}_{2} \otimes \mathbf{e}_{1}$, expression (28) reduces to

$$
\hat{I}^{(1)}=\frac{\bar{\gamma}^{2}}{(1-c)^{2}}+2 .
$$

Also, in this case, as $\omega \rightarrow \infty$, Eq. (30) simplifies to

$$
2 \bar{\lambda} \sin (\varphi)-\left(\bar{\lambda}^{2}-1\right) \sin [2(\varphi-\bar{\theta})]=0 .
$$

Now, recalling that for the special case of laminates the effective behavior can be computed exactly by making use of the fact that the fields are constant in the phases, it is straightforward to show that the exact result for the effective stored-energy function of the 
type of laminates considered here is given by $\widehat{W}^{I}(\overline{\mathbf{U}})=(\mathbf{1}-\mathbf{c}) \mathbf{g}^{(1)}\left(\overline{\mathbf{I}}^{(1)}\right)$, where $\bar{I}^{(1)}$ is the first invariant of the right Cauchy-Green deformation tensor associated with the exact average deformation gradient in the soft phase of the laminate, namely, $\bar{I}^{(1)}=\overline{\mathbf{F}}^{(1)} \cdot \overline{\mathbf{F}}^{(1)}$. Recognizing now that under simple shearing "parallel" to the layers the rotation of the rigid phase $\overline{\mathbf{R}}^{(2)}=\mathbf{I}$, the average deformation gradient in the soft phase may be computed exactly to yield $\overline{\mathbf{F}}^{(1)}=\mathbf{I}+\bar{\gamma} /(1-c) \mathbf{e}_{2} \otimes \mathbf{e}_{1}$. Thus, it can be deduced that $\hat{I}^{(1)}=\bar{I}^{(1)}$, so that the second-order estimate (27) recovers the exact result in the limit as $\omega \rightarrow \infty$. It is also a matter of straightforward algebra to show that for $\overline{\mathbf{F}}=\mathbf{I}+\bar{\gamma} \mathbf{e}_{2} \otimes \mathbf{e}_{1}$, expression (32) yields $\varphi=-\arcsin \left(\bar{\gamma} / \sqrt{4+\bar{\gamma}^{2}}\right)=-\bar{\psi}$, so that the angle of rotation of the rigid phase as predicted by the second-order estimate reduces to the exact result $\phi=0$.

On the other hand, for the isotropic case $(\omega=1)$, it is easy to show that $\hat{I}^{(1)}$ reduces to

$$
\hat{I}^{(1)}=\frac{1+2 c-4 c \bar{\lambda}+2 c^{2} \bar{\lambda}^{2}-4 c \bar{\lambda}^{3}+(1+2 c) \bar{\lambda}^{4}}{(1-c)^{2} \bar{\lambda}^{2}} .
$$

Note that $\hat{I}^{(1)}$ depends on the principal stretch $\bar{\lambda}$ (but not on the loading angle $\bar{\theta}$ ), so that the corresponding stored-energy function (27) is isotropic (in the plane of deformation). There are, unfortunately, no exact results available for the effective behavior of isotropic, rigidly reinforced, incompressible elastomers to which to compare (33). But it is easy to see from (30) that the second-order estimates predict that $\varphi$ should be exactly zero, so that the particles rotate precisely with the applied macroscopic rotation, i.e., $\phi=\bar{\psi}$, which is entirely consistent with the in-plane isotropic symmetry of the reinforcement and its distribution.

We conclude this section by remarking that we are not aware of any results in the literature concerning the rotation of rigid fibers in (incompressible) hyperelastic materials under finite-deformation conditions. Thus, relation (30), which appears to be physically consistent, at least from low to moderate concentration of fibers, and remarkably simple in character, is the first of its type.

\subsubsection{Application to rigidly reinforced, incompressible, Gent elastomers}

In this subsection, for definiteness, we specialize the general result (27) to the particular case of rigidly reinforced, incompressible, Gent elastomers. Thus, making use of relations (17) and (27), the effective stored-energy function for incompressible Gent elastomers reinforced with aligned rigid fibers of elliptical cross section, with volume fraction $c$ and aspect ratio $\omega,(\omega \geqslant 1)$, may be written as

$$
\widehat{W}^{I}(\overline{\mathbf{U}})=(c-1) \frac{\mu^{(1)} J_{m}}{2} \ln \left[1-\frac{\hat{I}^{(1)}-2}{J_{m}}\right],
$$

where $\hat{I}^{(1)}$ is given by (28). It then follows that the corresponding result for rigidly reinforced elastomers with incompressible Neo-Hookean matrix phases may be readily obtained upon taking the limit $J_{m} \rightarrow \infty$ in (34). The result reads as

$$
\widehat{W}^{I}(\overline{\mathbf{U}})=(1-c) \frac{\mu^{(1)}}{2}\left(\hat{I}^{(1)}-2\right) .
$$

The above expressions can be written more explicitly in the limit of in-plane isotropic symmetry of the reinforcement. In this context, $\hat{I}^{(1)}$ is given by (33) so that expression (34) 
may be shown to reduce to

$$
\widehat{W}^{I}(\overline{\mathbf{U}})=(c-1) \frac{\mu^{(1)} J_{m}}{2} \ln \left[1-\frac{(\bar{\lambda}-1)^{2}\left[(\bar{\lambda}+1)^{2}+2 c\left(\bar{\lambda}^{2}+1\right)\right]}{J_{m} \bar{\lambda}^{2}(1-c)^{2}}\right] .
$$

Similarly, (35) reduces to

$$
\widehat{W}^{I}(\overline{\mathbf{U}})=\frac{\mu^{(1)}(\bar{\lambda}-1)^{2}\left[(\bar{\lambda}+1)^{2}+2 c\left(\bar{\lambda}^{2}+1\right)\right]}{2 \bar{\lambda}^{2}(1-c)} .
$$

It is interesting to note that the effective stored-energy function (34) for reinforced Gent elastomers locks up when the condition $\hat{I}^{(1)}=J_{m}+2$ is satisfied. In order to get a representative notion of the behavior of this condition it is best to consider the simpler case of in-plane isotropic symmetry. For this case, it is straightforward to show that the stretch at which the material locks up is given by

$$
\bar{\lambda}_{\text {lock }}=p_{1}+\sqrt{\frac{1}{2}+p_{1}^{2}-p_{2}}+\sqrt{p_{1}\left(2 p_{1}+\sqrt{2+4 p_{1}^{2}-4 p_{2}}\right)-p_{2}-\frac{1}{2}}
$$

Here $p_{1}=c /(1+2 c)$ and $p_{2}=\left(4 c-2-(1-c)^{2} J_{m}\right) /(4+8 c)$. For comparison purposes, it is noted that the associated matrix phase material locks up at

$$
\bar{\lambda}_{\text {lock }}^{\text {matrix }}=\frac{\sqrt{2+J_{m}+\sqrt{J_{m}\left(4+J_{m}\right)}}}{\sqrt{2}}
$$

It is not difficult to check from (38) that $\bar{\lambda}_{\text {lock }}$ is a monotonically decreasing function of $c$ in the physical interval $c \in[0,1]$, taking the values $\bar{\lambda}_{\text {lock }}=\bar{\lambda}_{\text {lock }}^{\text {matrix }}$ at $c=0$ and $\bar{\lambda}_{\text {lock }}=1$ at $c=1$. In other words, reinforced Gent elastomers lock up at smaller finite stretches than the associated matrix phase materials. This is consistent with the fact that, on average, the deformation in the matrix phase of a rigidly reinforced material is larger than the macroscopic applied deformation (since the rigid phase does not deform), leading then to an overall smaller lock-up stretch.

As a final remark, it is noted that a result similar to (36) has been obtained (unpublished work) from an earlier version of the second-order estimate used in Lopez-Pamies and Ponte Castañeda (2004a) for the same type of Gent elastomeric composite. The two estimates, even though identical up to third order in the infinitesimal strain (i.e., up to $\left.O(\bar{\lambda}-1)^{3}\right)$, are significantly different for large values of the matrix lock-up parameter $J_{m}$ and high concentration of fibers $c$, at large deformations. This disparity is due to the difference in the limiting lock-up behavior of both estimates as $J_{m} \rightarrow \infty$. Indeed, unlike expression (38), which becomes unbounded as $J_{m} \rightarrow \infty$, the lock-up stretch computed from the earlier second-order estimate yields $\bar{\lambda}_{\text {lock }}=1 / c$ as $J_{m} \rightarrow \infty$. In essence, both types of estimates indicate that the presence of rigid particles enhances the lock-up effect, which is physically expected. However, we believe that the new predictions may be more realistic for composites with random microstructures, for which the addition of rigid particles would enhance the lock-up effect, provided that it is already present in the matrix phase. 


\subsection{Loss of strong ellipticity}

In this subsection, the specialization of the strong ellipticity condition (see Part I, Section 6)

$$
\widehat{K}_{i k} m_{i} m_{k}=\widehat{\mathscr{L}}_{i j k l} N_{j} N_{l} m_{i} m_{k}>0
$$

for all $\mathbf{m} \otimes \mathbf{N} \neq \mathbf{0}$ with $\widehat{\mathscr{L}}=\partial^{2} \widehat{W} / \partial \overline{\mathbf{F}}^{2}$, will be spelled out for the second-order estimates for the effective constitutive behavior of the compressible and incompressible reinforced elastomers developed in this section. To this end, and for simplicity in the incompressible case, it proves useful to choose the current configuration of the material as the reference state. Thus, recalling that (Chadwick and Ogden, 1971) $\widehat{\mathscr{L}}_{i j k l}=\bar{J} \bar{F}_{j r}^{-1} \bar{F}_{l s}^{-1} \widehat{\mathscr{L}}_{i r k s}^{c}$, where the superscript ' $c$ ' denotes evaluation in the current configuration, the strong ellipticity condition (40) may be rewritten as

$$
\widehat{K}_{i k}^{c} m_{i} m_{k}=\widehat{\mathscr{L}}_{i j k l}^{c} n_{j} n_{l} m_{i} m_{k}>0,
$$

for all $\mathbf{n} \otimes \mathbf{m} \neq \mathbf{0}$. Here, $\widehat{K}_{i k}^{c}=\widehat{\mathscr{L}}_{i j k l}^{c} n_{j} n_{l}$ is the acoustic tensor corresponding to the situation when the current and reference configuration coincide, and use has been made of the fact that $\bar{J}>0$. In this work, we are mainly interested in determining the boundary of the domain in deformation space, containing $\overline{\mathbf{F}}=\mathbf{I}$, at which strong ellipticity fails. (Recall that the type of reinforced elastomers considered here are characterized by strictly convex, and therefore strongly elliptic, effective stored-energy functions in the neighborhood of $\overline{\mathbf{F}}=\mathbf{I}$.) Then, it is clear that condition (41) will first cease to hold true away from $\overline{\mathbf{F}}=\mathbf{I}$ whenever the acoustic tensor $\widehat{\mathbf{K}}^{c}$ becomes singular. For plane-strain deformations, this amounts to the existence of real roots $n_{1} / n_{2}$ in the fourth-order polynomial equation

$$
\operatorname{det}\left[\widehat{\mathscr{L}}_{i 1 k 1}^{c}\left(\frac{n_{1}}{n_{2}}\right)^{2}+\left(\widehat{\mathscr{L}}_{i 1 k 2}^{c}+\widehat{\mathscr{L}}_{i 2 k 1}^{c}\right) \frac{n_{1}}{n_{2}}+\widehat{\mathscr{L}}_{i 2 k 2}^{c}\right]=0
$$

where $n_{1}$ and $n_{2}$ denote the direction cosines of the normal $\mathbf{n}$ to the characteristic direction in the deformed configuration. Explicit (but cumbersome) conditions on the components of the incremental modulus $\widehat{\mathscr{L}}^{c}$ may be written down in order for the quartic equation (42) to possess complex roots. However, in general, it is simpler to determine the onset of loss of strong ellipticity by monitoring the four roots of (42), which are known in closed form, along the loading path of interest, and detecting at which point at least one of these four roots becomes real. Once the real roots $n_{1} / n_{2}$ are detected, they can be substituted in expression (41) in order to compute the associated eigenvectors $\mathbf{m}$ corresponding to the zero eigenvalues of the acoustic tensor.

The loss-of-ellipticity condition (42) cannot be applied directly for incompressible reinforced elastomers. This is because the effective stored-energy function $\widehat{W}$ becomes unbounded for all deformations not satisfying the incompressibility constraint $\operatorname{det} \overline{\mathbf{F}}=1$, which implies that some of the components of the corresponding incremental modulus tensor $\widehat{\mathscr{L}}^{c}$ become unbounded as well. Consequently, condition (42) must be suitably adapted for incompressible elastomers. Noting that the incompressibility constraint $\operatorname{det} \overline{\mathbf{F}}=1$ implies that the vectors $\mathbf{n}$ and $\mathbf{m}$ in expression (41) must be such that $\mathbf{n} \cdot \mathbf{m}=\mathbf{0}$, it can be shown from (41) that, under plane-strain conditions, the loss of strong ellipticity is 
first attained away from $\overline{\mathbf{F}}=\mathbf{I}$, whenever the fourth-order polynomial equation

$$
\begin{aligned}
& \widehat{\mathscr{L}}_{2121}^{c}\left(\frac{n_{1}}{n_{2}}\right)^{4}-2\left(\widehat{\mathscr{L}}_{1121}^{c}-\widehat{\mathscr{L}}_{2221}^{c}\right)\left(\frac{n_{1}}{n_{2}}\right)^{3}+\left[\widehat{\mathscr{L}}_{1111}^{c}+\widehat{\mathscr{L}}_{2222}^{c}\right. \\
& \left.-2\left(\widehat{\mathscr{L}}_{1122}^{c}+\widehat{\mathscr{L}}_{1221}^{c}\right)\right]\left(\frac{n_{1}}{n_{2}}\right)^{2}+2\left(\widehat{\mathscr{L}}_{1112}^{c}-\widehat{\mathscr{L}}_{2212}^{c}\right) \frac{n_{1}}{n_{2}}+\widehat{\mathscr{L}}_{1212}^{c}=0
\end{aligned}
$$

admits one or more real roots $n_{1} / n_{2}$. It should be emphasized that the coefficients of the quartic equation (43), which correspond to projections of the acoustic tensor $\widehat{\mathbf{K}}^{c}$ onto the space of isochoric deformations, have finite values. Similar to the previous case of compressible materials, the loss of strong ellipticity of homogenized incompressible elastomers can be determined efficiently by monitoring the four roots of Eq. (43), which are available in closed-form, along the loading path of interest, and detecting at which point at least one of these four roots becomes real. For later use, it is helpful to record here the simplification of condition (43) for the situations in which the loading is aligned with the microstructure (i.e., without loss of generality, for $\bar{\theta}=0$ and $\bar{\theta}=\pi / 2$ ). Then, the odd terms disappear and we are led to

$$
\widehat{\mathscr{L}}_{2121}^{c}\left(\frac{n_{1}}{n_{2}}\right)^{4}+\left[\widehat{\mathscr{L}}_{1111}^{c}+\widehat{\mathscr{L}}_{2222}^{c}-2\left(\widehat{\mathscr{L}}_{1122}^{c}+\widehat{\mathscr{L}}_{1221}^{c}\right)\right]\left(\frac{n_{1}}{n_{2}}\right)^{2}+\widehat{\mathscr{L}}_{1212}^{c}=0 .
$$

Moreover, simple conditions (on the components of $\widehat{\mathscr{L}}^{c}$ ) may be written down in order for the fourth-order polynomial equation (44) to possess complex roots. Indeed, it is straightforward to show (see, e.g., Hill, 1979) that necessary and sufficient conditions for the quartic Eq. (44) to have complex roots are expressible as

$$
\begin{aligned}
& \text { (i) } \widehat{\mathscr{L}}_{1212}^{c}>0 \text {, (ii) } \widehat{\mathscr{L}}_{2121}^{c}>0, \\
& \text { (iii) } \sqrt{\widehat{\mathscr{L}}_{1212}^{c} \widehat{\mathscr{L}}_{2121}^{c}}-\left(\widehat{\mathscr{L}}_{1122}^{c}+\widehat{\mathscr{L}}_{1221}^{c}\right)+\frac{\widehat{\mathscr{L}}_{1111}^{c}+\widehat{\mathscr{L}}_{2222}^{c}}{2}>0 .
\end{aligned}
$$

Thus, under aligned plane-strain deformations, loss of strong ellipticity of homogenized, incompressible, reinforced elastomers will first take place at the point at which one of the inequalities in (45) fails to hold true.

Finally, it should be mentioned that the traces of the effective incremental modulus $\widehat{\mathscr{L}}^{c}$ that appear in the above conditions for the loss of strong ellipticity of incompressible, rigidly reinforced elastomers under plane-strain deformations may be conveniently written in terms of the effective stored-energy function $\breve{W}$, given by expression (27), and its first and second derivatives with respect to its arguments $\bar{\lambda}$ and $\bar{\theta}$. For brevity, the final expressions are not included here.

\section{Results for general plane-strain loading}

This section presents results associated with the second-order estimates for general plane-strain loading of fiber-reinforced elastomers with Gent and Neo-Hookean phases. Results are given for $\mu^{(1)}=1$ and various initial volume fractions, $c_{0}$, and aspect ratios, $\omega_{0}$, of the fibers, and were computed up to the point at which the effective incremental moduli were found to lose strong ellipticity, or truncated at some sufficiently large strain if no such loss was found. For clarity, the points at which loss of strong ellipticity is 
encountered are denoted with the symbol $\circ$ in the plots. The results and discussion for pure shear loading of (in-plane) isotropic (i.e., $\omega=1$ ), rigidly reinforced, incompressible, Gent elastomers are presented first. They are followed by the results for pure shear loading of compressible and incompressible Gent and Neo-Hookean elastomers reinforced with fibers of elliptical cross section (i.e., $\omega>1$ ). Finally, results for simple shear of rigidly reinforced, incompressible, Neo-Hookean elastomers are discussed. The idea behind the choice of these results is to bring out the effect of the microstructure evolution, which depends critically on the boundary conditions, on the overall response and stability of the material.

\subsection{Pure shear loading: circular rigid fibers and incompressible matrix}

Fig. 2 presents the effective behavior as predicted by the second-order method for an incompressible Gent elastomer $\left(\kappa^{(1)} \rightarrow \infty\right)$ reinforced with rigid fibers of circular cross section $(\omega=1)$ under pure shear loading $\left(\bar{\lambda}_{1}=\bar{\lambda}_{2}^{-1}=\bar{\lambda}\right)$. Results are shown for fiber concentrations of $10 \%, 20 \%$ and $30 \%$, and a value of the matrix lock-up parameter $J_{m}=50$, as a function of the macroscopic stretch $\bar{\lambda}$. Part (a) shows the effective storedenergy function, and part (b), the associated stress $\bar{S}=\mathrm{d} \widehat{W} / \mathrm{d} \bar{\lambda}$. Note that the closed-form expression for the effective stored-energy function shown in Fig. 2(a) is given by (36). A major observation that can be made from Fig. 2 is the increasing reinforcement effect of the elastomeric matrix with the addition of rigid fibers, which is consistent with experimental observations. It is also interesting to remark that the stretch at which the elastomeric composite locks up depends very strongly on the concentration of fibers. Indeed, it is observed that the composite locks up at smaller stretches with increasing values of $c$, as anticipated in the previous section.

Fig. 3 shows corresponding plots for the effective behavior of an incompressible Gent elastomer reinforced with $30 \%$ of rigid fibers of circular cross section under pure shear
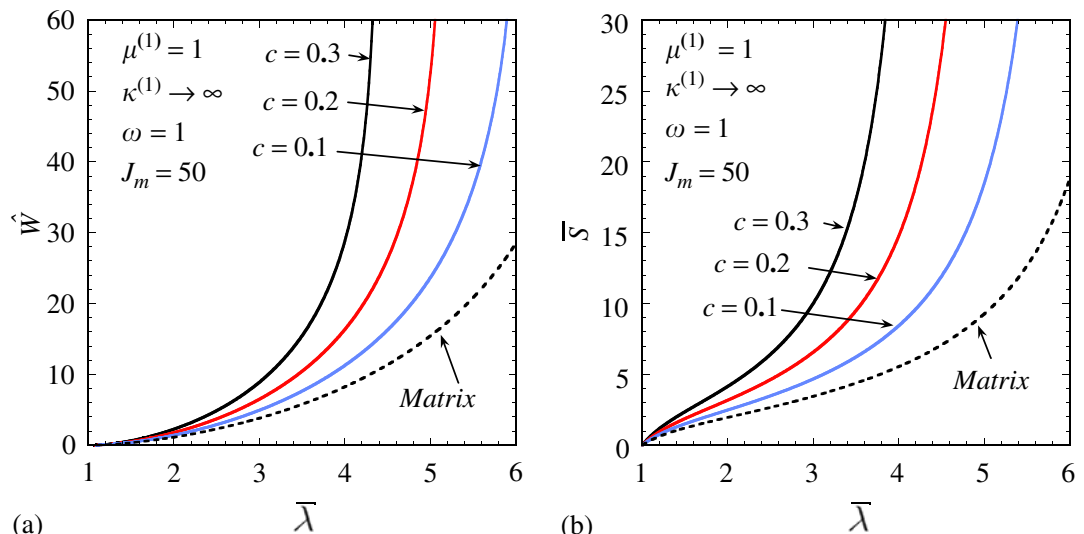

Fig. 2. Second-order estimates for the effective behavior of elastomers reinforced with rigid fibers of circular cross section subjected to pure shear loading. The results correspond to an incompressible Gent matrix phase with given matrix lock-up parameter $J_{m}=50$ and various values of the fiber concentration $c$, and are shown as a function of the principal macroscopic stretch $\bar{\lambda}$. (a) The effective stored-energy function $\widehat{W}$. (b) The corresponding stress $\bar{S}=\mathrm{d} \widehat{W} / \mathrm{d} \bar{\lambda}$. 

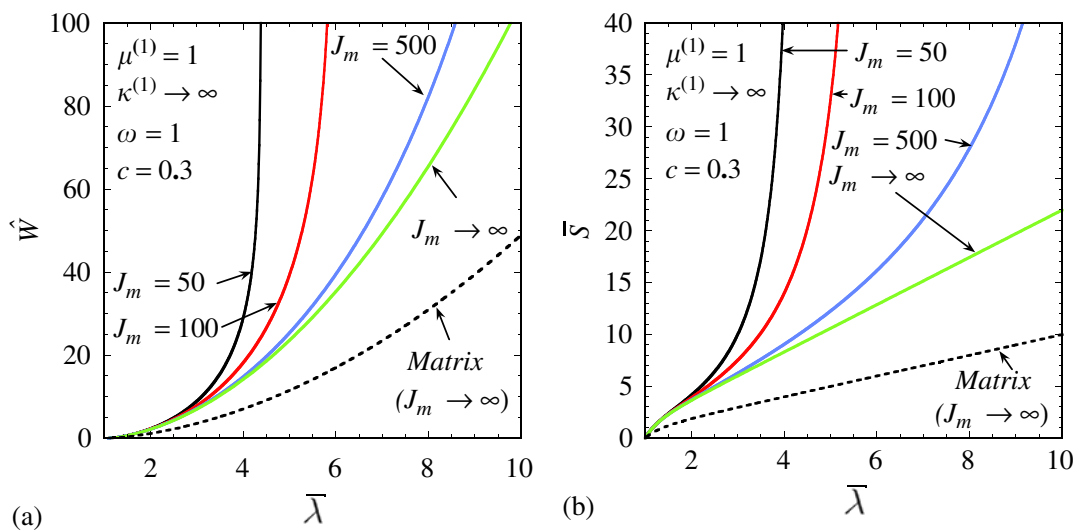

Fig. 3. Second-order estimates for the effective behavior of elastomers reinforced with rigid fibers of circular cross section subjected to pure shear loading. The results correspond to an incompressible Gent matrix phase with $c=30 \%$ and various values of the matrix lock-up parameter $J_{m}$, and are shown as a function of the principal macroscopic stretch $\bar{\lambda}$. (a) The effective stored-energy function $\widehat{W}$. (b) The corresponding stress $\bar{S}=\mathrm{d} \widehat{W} / \mathrm{d} \bar{\lambda}$.

loading for values of the matrix lock-up parameter $J_{m}=50,100,500$, and $J_{m} \rightarrow \infty$, as a function of the macroscopic stretch $\bar{\lambda}$. Part (a) shows the effective stored-energy function, and part (b), the associated stress $\bar{S}=\mathrm{d} \widehat{W} / \mathrm{d} \bar{\lambda}$. It is observed from Fig. 3 that the overall response of the reinforced elastomer is strongly dependent on the matrix lock-up parameter $J_{m}$, which is not surprising since the response of the matrix itself is also highly dependent on $J_{m}$. It is further noted from this figure that the second-order estimate for the effective behavior of a rigidly reinforced Neo-Hookean elastomer (i.e., $J_{m} \rightarrow \infty$ ) does not exhibit lock-up at finite stretch, as already discussed in the context of Eq. (38). This is in contrast to earlier results by Ponte Castañeda and Tiberio (2000) and Lopez-Pamies and Ponte Castañeda (2004a) using earlier versions of the second-order method, where lock-up was found to be attained at $\bar{\lambda}=1 / c$ for reinforced Neo-Hookean elastomers. For the reasons stated in Section 3.2.4, we believe that the new predictions are more accurate in the present context of composites with random microstructures.

Fig. 4 provides plots associated with the results shown in Figs. 2 and 3 for the macroscopic stretch $\bar{\lambda}_{\text {lock }}$ at which a rigidly reinforced, incompressible, Gent elastomer locks up. Part (a) shows $\bar{\lambda}_{\text {lock }}$ for values of the matrix lock-up parameter $J_{m}=50,100$, and 500 , as a function of the fiber concentration $c$. Part (b) shows $\bar{\lambda}_{\text {lock }}$ for fiber concentrations of $0 \%, 10 \%, 20 \%$, and $30 \%$, as a function of $J_{m}$. Recall that the closed-form expression for the stretch $\bar{\lambda}_{\text {lock }}$ shown in Fig. 4 is given by (38). The key point to be drawn from Fig. 4 is that the elastomeric composite $(c>0)$ locks up at a smaller stretch than the corresponding matrix phase $(c=0)$. In fact, Fig. 4(a) shows that $\bar{\lambda}_{\text {lock }}$ decreases monotonically from the lock-up of the matrix phase $\left(\bar{\lambda}_{\text {lock }}=\bar{\lambda}_{\text {lock }}^{\text {matrix }}\right.$ at $\left.c=0\right)$ to that of the rigid phase $\left(\bar{\lambda}_{\text {lock }}=1\right.$ at $\left.c=1\right)$ with increasing $c$, as previously discussed. In addition, Fig. 4(b) shows that the influence of the matrix lock-up parameter $J_{m}$ on $\bar{\lambda}_{\text {lock }}$ weakens monotonically as $J_{m}$ increases.

Finally, it is important to stress from the above results that (in-plane) isotropic $(\omega=1)$, rigidly reinforced, incompressible, Gent elastomers, which are strongly elliptic in the pure state, remain strongly elliptic for all deformations. 

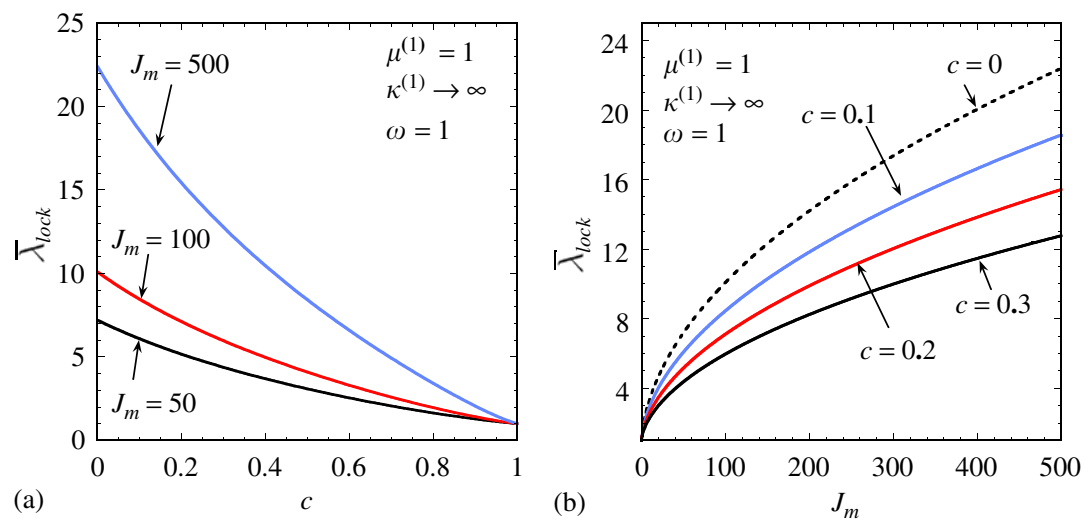

Fig. 4. Second-order estimates for the macroscopic stretch $\bar{\lambda}_{\text {lock }}$ at which an (in-plane) isotropic rigidly reinforced incompressible Gent elastomer locks up. (a) $\bar{\lambda}_{\text {lock }}$ as a function of fiber concentration $c$ for various values of the matrix lock-up parameter $J_{m}$. (b) $\bar{\lambda}_{\text {lock }}$ as a function of $J_{m}$ for various values of $c$.
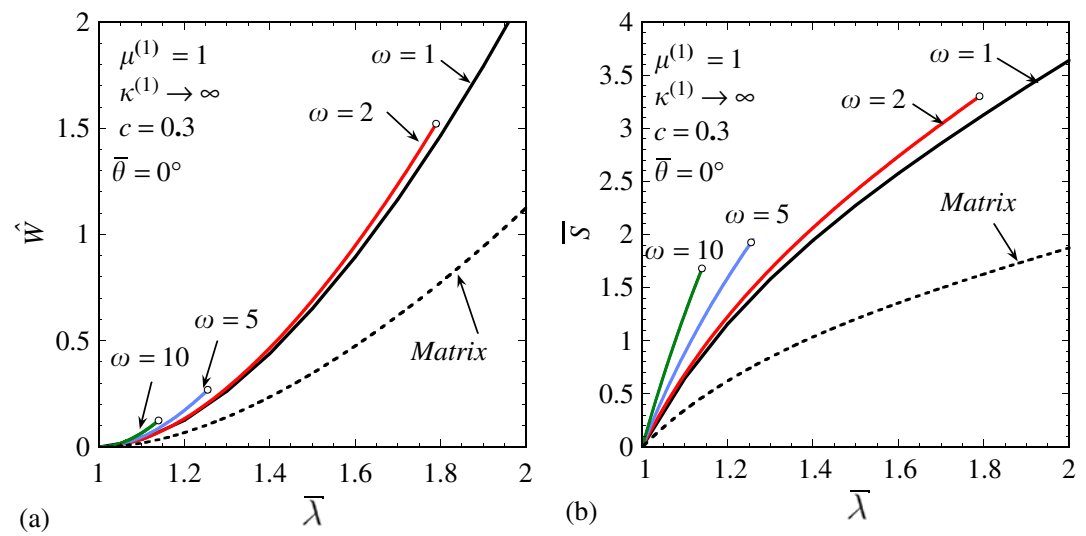

Fig. 5. Second-order estimates for the effective behavior of rigidly reinforced elastomers subjected to aligned pure shear loading $\left(\bar{\theta}=0^{\circ}\right)$. The results correspond to an incompressible, Neo-Hookean matrix phase with given fiber concentration $c=30 \%$, and various values of the fiber aspect ratio $\omega$, and are shown as a function of the principal macroscopic stretch $\bar{\lambda}$. (a) The effective stored-energy function $\widehat{W}$. (b) The corresponding stress $\bar{S}=\mathrm{d} \widehat{W} / \mathrm{d} \bar{\lambda}$.

\subsection{Aligned pure shear loading: rigid fibers and incompressible matrix}

Fig. 5 presents the effective behavior as predicted by the second-order method for an incompressible, Neo-Hookean elastomer reinforced with rigid fibers of elliptical cross section under aligned pure shear loading with $\bar{\theta}=0^{\circ}$, i.e., compression along the longest in-plane axis of the fibers. Results are shown for fiber aspect ratios of 1,2, 5, and 10, and fiber concentration of $30 \%$, as a function of the macroscopic stretch $\bar{\lambda}$. Part (a) gives the effective stored-energy function, and part (b), the associated stress $\bar{S}=\mathrm{d} \widehat{W} / \mathrm{d} \bar{\lambda}$. Note that expression (35) for the effective stored-energy function shown in Fig. 5(a) simplifies even further by recognizing, from (30), that for aligned pure shear loadings $\varphi=0^{\circ}$. Also note that for this type of loading, the macroscopic rotation tensor $\overline{\mathbf{R}}=\mathbf{I}$, which together with the fact that $\varphi=0^{\circ}$, implies that the fibers do not rotate, namely, $\phi=0^{\circ}$. Similar to the 
case of pure shear loading of in-plane, isotropic, rigidly reinforced elastomers, the results shown in Fig. 5 exhibit a significant reinforcement effect undergone by the elastomeric matrix with the addition of rigid fibers. The reinforcement is more pronounced for higher values of the aspect ratio. This is consistent with the fact that in the limit $\omega \rightarrow \infty$ (as the microstructure tends to a laminate) the material becomes rigid under the given loading conditions. Moreover, Fig. 5 shows that the second-order method predicts loss of strong ellipticity of the homogenized behavior of reinforced elastomers under aligned pure shear loading with $\bar{\theta}=0^{\circ}$ for fiber aspect ratios $\omega>1$. More particularly, it is observed that loss of strong ellipticity takes place at smaller stretches for higher $\omega$. That is, under the type of deformation considered here, the composite stiffens, but also becomes more unstable with increasing values of the aspect ratio of the fibers.

Fig. 6 provides plots associated with the results shown in Fig. 5 for the critical stretch $\bar{\lambda}_{\text {crit }}$ at which the loss of strong ellipticity occurs for the homogenized behavior of rigidly reinforced, incompressible, Neo-Hookean elastomers. Part (a) shows $\bar{\lambda}_{\text {crit }}$ for concentration of fibers of $1 \%, 10 \%, 20 \%$, and $30 \%$, as a function of the aspect ratio $\omega$, and part (b), $\bar{\lambda}_{\text {crit }}$ for aspect ratios of 2,5 , and 10 , as a function of the concentration of fibers $c$. It is seen from Fig. 6(a) that, as already mentioned in the context of Fig. 5, elastomers reinforced with fibers of higher aspect ratio lose strong ellipticity at smaller stretches. Another key point to be drawn from Fig. 6 (a) is that $\bar{\lambda}_{\text {crit }}$ has a vertical asymptote at $\omega=1$. This entails that (in-plane) isotropic, rigidly reinforced, incompressible, Neo-Hookean elastomers remain strongly elliptic under all deformations, in agreement with the results shown in the preceding subsection. However, as soon as the isotropic symmetry is perturbed, the homogenized material loses strong ellipticity at some large, but finite, stretch. Note that $\bar{\lambda}_{\text {crit }} \rightarrow 1$ as $\omega \rightarrow \infty$, in agreement with the exact result for the corresponding laminate. Also note that $\bar{\lambda}_{\text {crit }}$ is smaller for higher values of the concentration of fibers, so that reinforced elastomers with a higher content of fibers are more unstable. This point is more clearly illustrated by Fig. 6(b). In addition, we remark from Fig. $6(\mathrm{~b})$ that $\bar{\lambda}_{\text {crit }}$ tends to a finite value as $c \rightarrow 0$. In this connection, recall that the Neo-Hookean elastomer utilized here for the matrix phase is a strongly elliptic material for all deformations. This implies
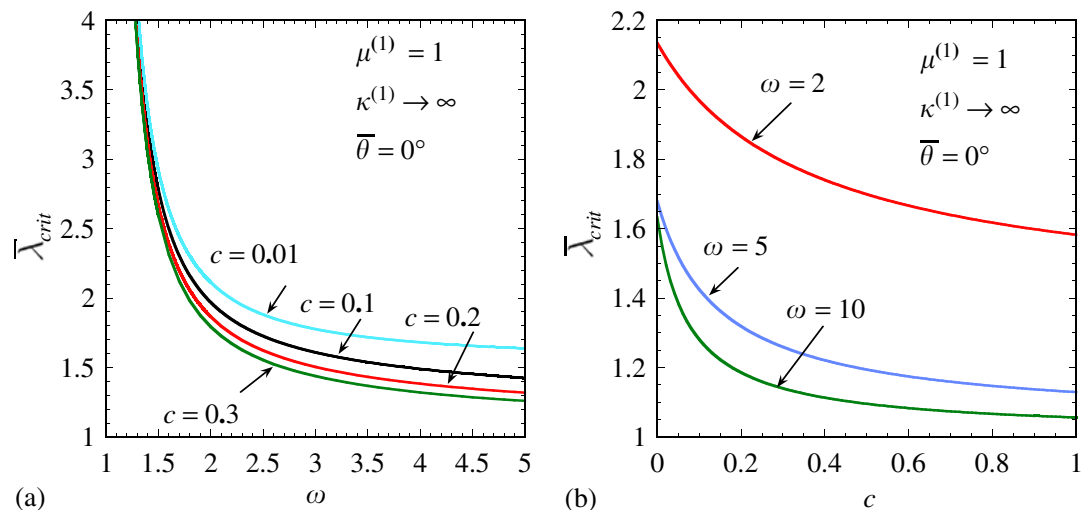

Fig. 6. Aligned pure shear loading $\left(\bar{\theta}=0^{\circ}\right)$ of a rigidly reinforced, incompressible, Neo-Hookean elastomer. (a) The critical stretch $\bar{\lambda}_{\text {crit }}$ at which loss of strong ellipticity of the homogenized elastomer takes place for various fiber concentrations as a function of the aspect ratio of the fibers $\omega$. (b) The critical stretch $\bar{\lambda}_{\text {crit }}$ for various aspect ratios as a function of the concentration of fibers $c$. 
that $\bar{\lambda}_{\text {crit }}$ has a singularity at $c=0$, namely, $\bar{\lambda}_{\text {crit }}=\infty$ at $c=0$, but $\bar{\lambda}_{\text {crit }} \rightarrow \bar{\lambda}_{\text {crit }}^{0}$ as $c \rightarrow 0$, where $0<\bar{\lambda}_{\text {crit }}^{0}<\infty$. Physically, this result suggests that the addition of even a small proportion of aligned, elliptical, rigid fibers can have a dramatic effect on the overall stability of an incompressible, strongly elliptic elastomer at some sufficiently large, but finite, compressive stretch along the longest in-plane axes of the fibers.

Finally, it is important to note that it was through failure of the condition (i) in (45) that strong ellipticity was systematically lost in the results shown in Fig. 6. Within the context of condition (44), the fact of having the incremental effective modulus $\widehat{\mathscr{L}}_{1212}^{c}$ vanish implies that the normal to the characteristic direction in the deformed configuration is given by $\mathbf{n}=\mathbf{e}_{2}$, so that, by virtue of the incompressibility constraint, $\mathbf{m}=\mathbf{e}_{1}$. That is, the homogenized material may develop localized shear deformations in the plane determined by the normal $\mathbf{e}_{2}$, and in the direction $\mathbf{e}_{1}$. Making contact with the microstructure, the condition $\widehat{\mathscr{L}}_{1212}^{c}=0$, under the given type of loading, would correspond to the possible flopping of the fibers, which is a physically plausible instability mechanism for these materials.

Fig. 7 presents additional results for the critical stretch $\bar{\lambda}_{\text {crit }}$ for rigidly reinforced elastomers, with a fiber concentration of $30 \%$, subjected to aligned pure shear loading with $\bar{\theta}=0^{\circ}$, as a function of the aspect ratio $\omega$. Part (a) gives results for incompressible, Gent elastomers with matrix lock-up parameters $J_{m}=50$ and 100 , and part (b), for compressible, Neo-Hookean elastomers with bulk moduli $\kappa^{(1)}=11,101$, and $\kappa^{(1)} \rightarrow \infty$. From Fig. 7(a) it is discerned that the matrix lock-up parameter $J_{m}$ has essentially no effect on the onset of loss of strong ellipticity of rigidly reinforced, incompressible, Gent elastomers. As a matter of fact, $\bar{\lambda}_{\text {crit }}$ is completely independent of $J_{m}$ up to the point at which the material locks up. (Note that the stretch at which lock-up takes place has been included in Fig. 7(a) for reference purposes.) The main point that can be drawn from Fig. 7(b) is that the loss of strong ellipticity of rigidly reinforced elastomers is very much dependent on the compressibility of the matrix phase. Indeed, it is found from this figure
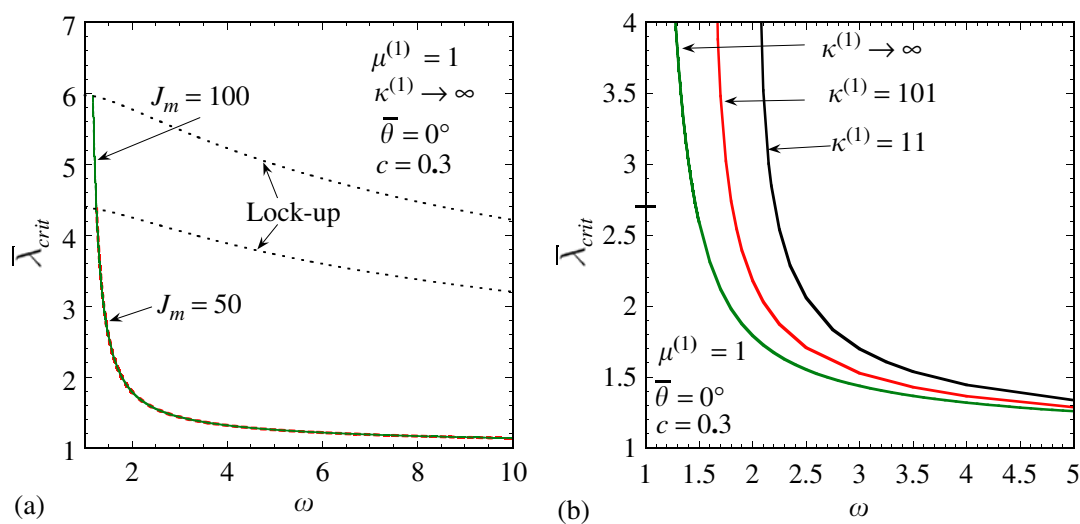

Fig. 7. Aligned pure shear loading $\left(\bar{\theta}=0^{\circ}\right)$ of rigidly reinforced elastomers. (a) The critical stretch $\bar{\lambda}_{\text {crit }}$ at which loss of strong ellipticity of a rigidly reinforced, incompressible, Gent elastomer takes place for various values of the lock-up parameter $J_{m}$ as a function of the aspect ratio of the fibers $\omega$. (b) The corresponding critical stretch $\bar{\lambda}_{\text {crit }}$ for a rigidly reinforced, compressible, Neo-Hookean elastomer, at various values of the bulk modulus $\kappa^{(1)}$, as a function of the aspect ratio of the fibers $\omega$. 
that rigidly reinforced, Neo-Hookean elastomers become more unstable with increasing incompressibility of the matrix phase (i.e., for higher values of $\kappa^{(1)}$ ). This trend is consistent with the numerical results obtained by Triantafyllidis et al. (2005) for the loss of ellipticity of reinforced elastomers with periodic microstructures. It should be mentioned that, similar to the results shown in Fig. 6, all the results for loss of strong ellipticity displayed in Fig. 7 are due to the vanishing of the effective incremental shear modulus $\widehat{\mathscr{L}}_{1212}^{c}$, which, again, corresponds to a possible flopping-type instability.

\subsection{Pure shear loading at an angle: rigid fibers and incompressible matrix}

Fig. 8 presents the effective behavior, as predicted by second-order estimate (35), for an incompressible, Neo-Hookean elastomer reinforced with rigid fibers of elliptical cross section under pure shear loading at the fixed angle $\bar{\theta}=20^{\circ}$. Results are shown for a fiber concentration of $30 \%$ as a function of the macroscopic stretch $\bar{\lambda}$. Part (a) gives the effective stress $\bar{S}=\mathrm{d} \widehat{W} / \mathrm{d} \bar{\lambda}$ for fiber aspect ratios of $\omega=1,2,5,10$, and part (b), the average angle of rotation of the fibers $\phi$, for fiber aspect ratios of $\omega=1,1.1,1.5,2,5$, and $\omega \rightarrow \infty$. Note that for the given type of loading, the macroscopic rotation tensor $\overline{\mathbf{R}}$ is exactly equal to the identity so that the angle defined by Eq. (30), $\varphi$, corresponds to the angle of rotation of the fibers, namely, $\phi=\varphi$. Similar to the previous cases, Fig. 8(a) shows a significant reinforcement effect of the matrix phase with the addition of rigid fibers. However, unlike the results for aligned pure shear loading with $\bar{\theta}=0^{\circ}$, where higher values of the aspect ratio of the fibers were found to consistently provide a higher reinforcement effect, higher aspect ratios of the fibers lead here to a stiffer behavior of the composite only for small deformations, whereas for large stretches the opposite is true. Furthermore, only the elastomer with $\omega=10$ in Fig. 8(a) is found to lose strong ellipticity. This behavior is also different from what it was observed for aligned pure shear loading with $\bar{\theta}=0^{\circ}$, where the break of isotropic symmetry (i.e., $\omega>1$ ) was shown to lead systematically to loss of strong ellipticity of the homogenized elastomer at some finite stretch. The above-mentioned disparities will be shown shortly to be linked to the evolution of the microstructure.
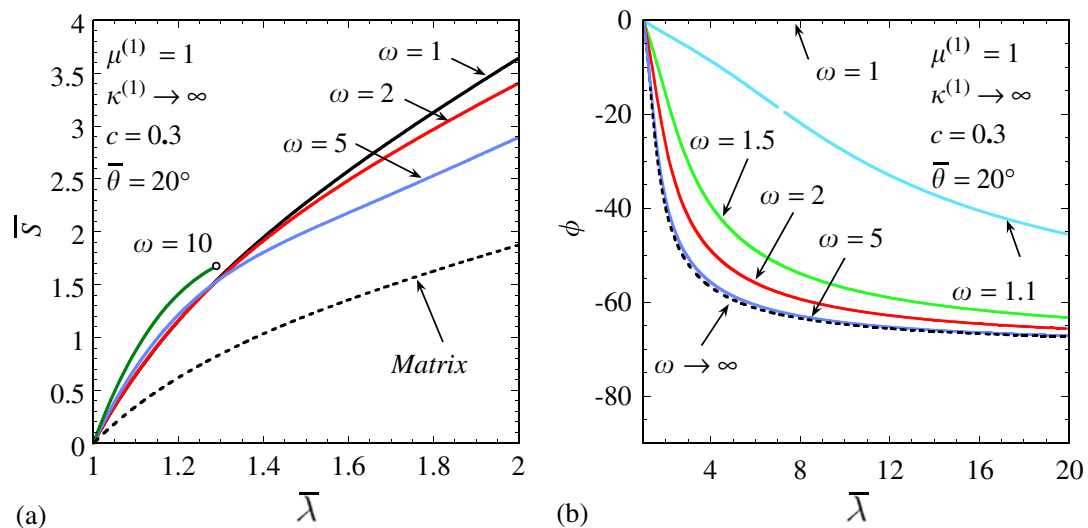

Fig. 8. Second-order estimates for the effective behavior of rigidly reinforced elastomer subjected to pure shear loading at a fixed angle $\left(\bar{\theta}=20^{\circ}\right)$. The results correspond to an incompressible, Neo-Hookean, matrix phase with given fiber concentration $c=30 \%$, and various values of the fiber aspect ratio $\omega$, and are shown as a function of the principal macroscopic stretch $\bar{\lambda}$. (a) The effective stress $\bar{S}=\mathrm{d} \widehat{W} / \mathrm{d} \bar{\lambda}$. (b) The angle of rotation of the fibers $\phi$. 
Fig. 8(b) shows that circular fibers $(\omega=1)$ do not rotate under pure shear at a fixed angle, as previously discussed. On the other hand, elliptical fibers $(\omega>1)$ do rotate clockwise (with respect to the fixed frame of reference) under the present loading conditions, aligning their longest in-plane principal axes with the tensile axis, that is, for this case, $\varphi \rightarrow-70^{\circ}$ as $\bar{\lambda} \rightarrow \infty$. It is also interesting to observe that fibers with higher aspect ratio rotate faster. In this regard, it is noted that the angle of rotation for the limiting case $\omega \rightarrow \infty$ has been included in Fig. 8 for reference purposes. However, it must be recalled that the composite behaves rigidly for $\omega \rightarrow \infty$ under the given loading conditions. In view of the results for the overall constitutive response shown in Fig. 8(a) and the evolution of the associated underlying microstructure shown in Fig. 8(b), it is inferred that the rotation of the fibers constitutes, in the present context, a softening mechanism. Physically, the rigid rotations of the fibers serve to "accommodate" part of the applied macroscopic loading, which hinders the hardening of the matrix phase of the material. Given that fibers with larger aspect ratios rotate faster, they are able to "accommodate" a larger amount of the applied macroscopic deformation. This leads to a stronger softening effect which is consistent with the results shown in Fig. 8(a) at large stretches.

Fig. 9 provides plots for the effective behavior as predicted by the second-order method for an incompressible, Neo-Hookean elastomer reinforced with rigid fibers of elliptical cross section under pure shear loading at the fixed angles $\bar{\theta}=0^{\circ}, 5^{\circ}, 30^{\circ}, 50^{\circ}, 70^{\circ}$, and $90^{\circ}$. Results are shown for a fiber aspect ratio of 2 and fiber concentration of $30 \%$, as a function of the macroscopic stretch $\bar{\lambda}$. Part (a) shows the effective stress $\bar{S}=\mathrm{d} \widehat{W} / \mathrm{d} \bar{\lambda}$, and part (b), the average angle of rotation of the fibers $\phi$. As for the preceding results, $\overline{\mathbf{R}}=\mathbf{I}$, so that $\phi=\varphi$. A key observation that can be made from Fig. 9(a), besides the clear reinforcement effect undergone by the matrix phase with the addition of rigid fibers, is that for large deformations, except for the case with $\bar{\theta}=0^{\circ}$, the reinforced elastomer consistently shows a stiffer response for higher angles of loading, with the stiffest behavior being attained at $\bar{\theta}=90^{\circ}$. For exactly $\bar{\theta}=0^{\circ}$, the effective response coincides with that for
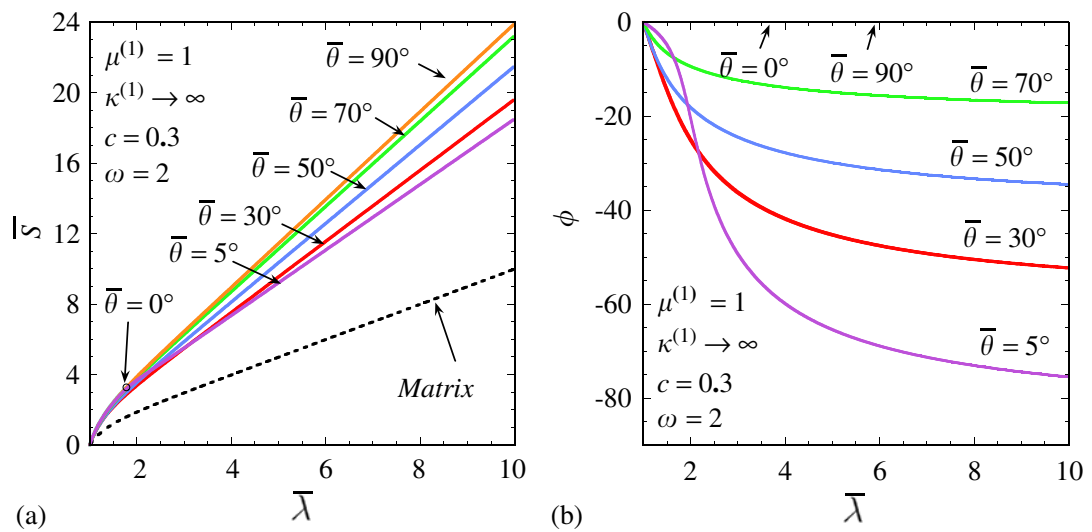

Fig. 9. Second-order estimates for the effective behavior of rigidly reinforced elastomer subjected to pure shear loading at various angles $\bar{\theta}$ (in the large deformation regime). The results correspond to an incompressible, NeoHookean matrix phase with given fiber concentration $c=30 \%$ and aspect ratio $\omega=2$, and are shown as a function of the principal macroscopic stretch $\bar{\lambda}$. (a) The effective stress $\bar{S}=\mathrm{d} \widehat{W} / \mathrm{d} \bar{\lambda}$. (b) The average angle of rotation of the fibers $\phi$. 
$\bar{\theta}=90^{\circ}$ for all stretches. This can be easily verified from (28) by noting, from (30), that for aligned loadings (i.e., for $\bar{\theta}=0^{\circ}$ and $\bar{\theta}=90^{\circ}$ ) $\varphi$ is exactly equal to $0^{\circ}$, as it has already been pointed out. It is further noted from Fig. 9(a) that loss of strong ellipticity of the homogenized elastomer takes place for pure shear with $\bar{\theta}=0^{\circ}$, which corresponds to compression along the longest in-plane axes of the fibers. On the other hand, for the pure shear loading with $\bar{\theta}=90^{\circ}$, which corresponds to tension along the longest in-plane axes of the fibers, the homogenized elastomer does not lose strong ellipticity. This result might seem inconsistent at first. However, it should be recalled that the overall behavior of the material is anisotropic, and that even though the stress-stretch relations (in the loading direction) are identical for both pure shear deformations with $\bar{\theta}=0^{\circ}$ and $\bar{\theta}=90^{\circ}$, the corresponding incremental moduli are in fact different. In this regard, it is evoked that the loss of strong ellipticity of the homogenized elastomer under pure shear with $\bar{\theta}=0^{\circ}$ is due to the fact that $\widehat{\mathscr{L}}_{1212}^{c}=0$ at $\bar{\lambda}_{\text {crit }}$, which physically is associated with a possible flopping instability of the fibers. On the other hand, for the case of $\bar{\theta}=90^{\circ}$, the effective shear modulus $\widehat{\mathscr{L}}_{1212}^{c}$ is not only positive, but it increases with the applied stretch.

Fig. 9(b) shows that the elliptical fibers with $\omega=2$ do rotate clockwise (with respect to the fixed frame of reference) aligning their longest in-plane principal axes with the principal direction of tensile loading (namely, $\phi \rightarrow \bar{\theta}-90^{\circ}$ as $\bar{\lambda} \rightarrow \infty$ ), for all loadings, except at $\bar{\theta}=0^{\circ}$, for which, again, the fibers do not rotate, but instead, remain fixed with their longest in-plane principal axes aligned with the principal direction of compressive loading. In essence, for aligned pure shear loadings (i.e., $\bar{\theta}=0^{\circ}$ and $\bar{\theta}=90^{\circ}$ ) there is no evolution of the orientation of the fibers. On the contrary, for "misaligned" deformations, the fibers do undergo a total rotation equal to the complementary angle of $\bar{\theta}$ as $\bar{\lambda} \rightarrow \infty$, in agreement with the discussion of the large-deformation behavior of Eq. (30) in Section 3.2.2. This entails that reinforced elastomers deformed at smaller loading angles $\bar{\theta}$ are able to "accommodate" a larger portion of the applied macroscopic loading by rigid rotation of the underlying fibers, which has already been identified as a softening mechanism. This is entirely consistent with the fact that the effective stress-stretch relations shown in Fig. 9(a) are systematically stiffer for higher $\bar{\theta}$ (with the exception of $\bar{\theta}=0^{\circ}$ ), in the finite-deformation regime.

Fig. 10 provides plots for a blow up in the small deformation regime of some of the results shown in Fig. 9. Fig. 10(a) corresponds to a blow up of Fig. 9(a) for the cases of $\bar{\theta}=0^{\circ}, 30^{\circ}, 60^{\circ}$, and $90^{\circ}$. It can be seen from this figure that loadings at complementary angles produce an identical effective response of the material in the small deformation regime, in accordance with the linear theory. For sufficiently large deformations, the effective responses at complementary angles deviate from each other, due to the difference in the evolution of the microstructure, as already discussed. Fig. 10(b) corresponds to a blow up of Fig. 9(b) for the cases of $\bar{\theta}=0^{\circ}$ and $\bar{\theta}=5^{\circ}$ in which the results for $\bar{\theta}=$ $0.1^{\circ}, 0.5^{\circ}, 1^{\circ}, 2^{\circ}$, and $3^{\circ}$ have been included in order to aid the discussion. In the previous section (as well as in some of the preceding results discussed in this section), it was observed that the fibers in the type of rigidly reinforced elastomers studied here do not rotate when subjected to pure shear deformations at exactly $\bar{\theta}=0^{\circ}$. However, an infinitesimal misalignment of this loading angle was shown to result into a $90^{\circ}$ rotation of the fibers as the applied stretch $\bar{\lambda}$ was increased. In this connection, Fig. 10(b) clearly shows that loadings at small angles $\bar{\theta}$ can lead to large rotations of the fibers. Interestingly, this figure shows that for relatively small loading angles $\bar{\theta}$, the corresponding rotation of the fibers develops a highly nonlinear evolution as a function of the applied macroscopic 

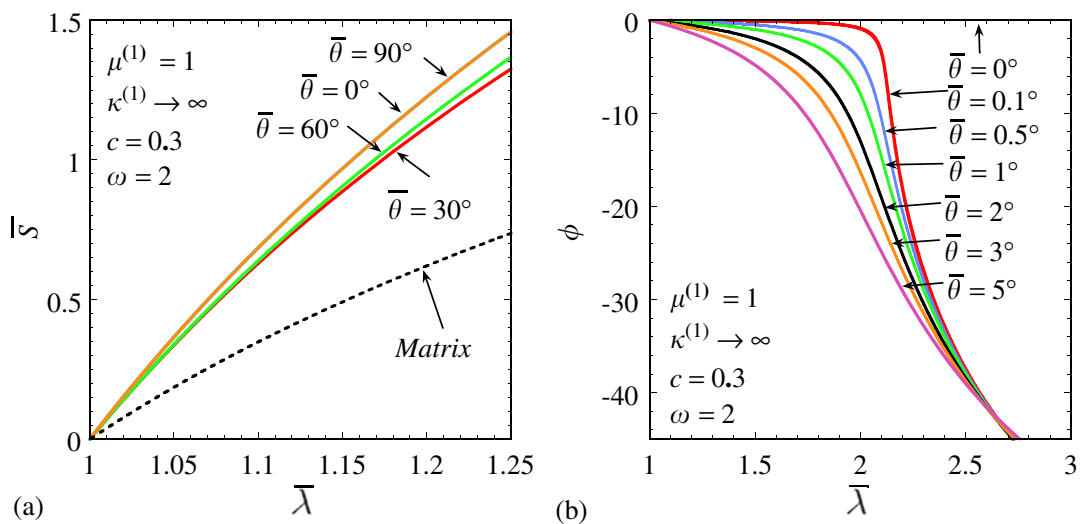

Fig. 10. Second-order estimates for the effective behavior of rigidly reinforced elastomer subjected to pure shear loading at various angles $\bar{\theta}$ (in the small deformation regime). The results correspond to an incompressible, NeoHookean matrix phase with given fiber concentration $c=30 \%$ and aspect ratio $\omega=2$, and are shown as a function of the principal macroscopic stretch $\bar{\lambda}$. (a) The effective stress $\bar{S}=\mathrm{d} \widehat{W} / \mathrm{d} \bar{\lambda}$. (b) The average angle of rotation of the fibers $\phi$.
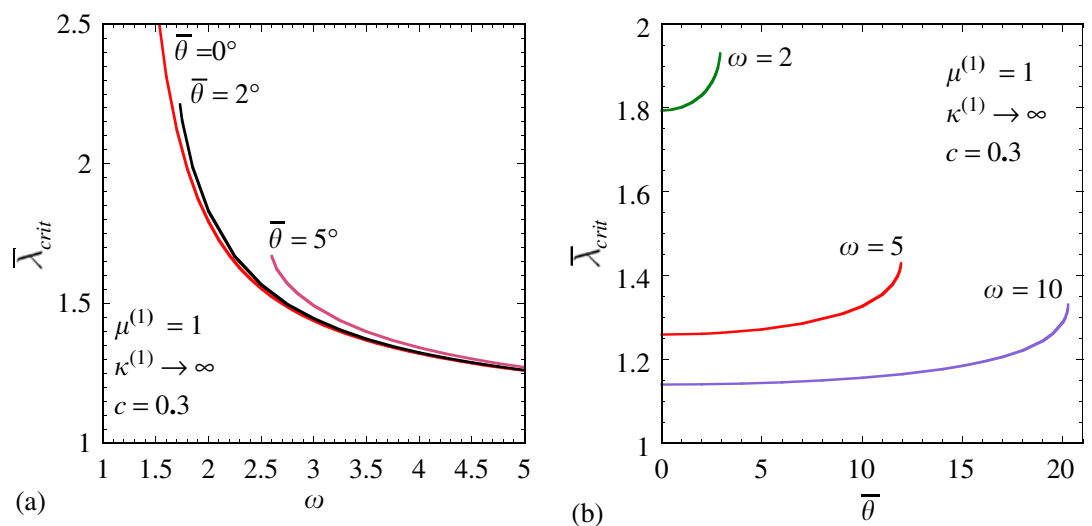

Fig. 11. Pure shear loading of rigidly reinforced elastomers. (a) The critical stretch $\bar{\lambda}_{\text {crit }}$ at which loss of strong ellipticity of a rigidly reinforced, incompressible, Neo-Hookean elastomer takes place for various loading angles $\bar{\theta}$, as a function of the aspect ratio of the fibers $\omega$. (b) The corresponding results for $\bar{\lambda}_{\text {crit }}$ for various values of the aspect ratio of the fiber as a function of the angle of loading $\bar{\theta}$.

stretch $\bar{\lambda}$. Indeed, as $\bar{\theta}$ approaches $0^{\circ}, \phi$ remains small initially as $\bar{\lambda}$ increases up to certain finite stretch at which it undergoes a dramatic increase. In essence, by making use of the established fact that larger fiber rotations potentially lead to a softer overall constitutive response, it is seen that a slight misalignment (about $\bar{\theta}=0^{\circ}$ ) in the applied loading can result into a drastically different, much softer, effective response of the material at large deformations. This is entirely consistent with the emergence of flopping-type instabilities at $\theta=0^{\circ}$.

Fig. 11 shows the effect of the loading angle $\bar{\theta}$ on the critical stretch $\bar{\lambda}_{\text {crit }}$ for rigidly reinforced, incompressible, Neo-Hookean elastomers subjected to pure shear. Results are shown for a fiber concentration of $30 \%$. Part (a) gives $\bar{\lambda}_{\text {crit }}$ for loading angles of $\bar{\theta}=0^{\circ}, 2^{\circ}$, 
and $5^{\circ}$, as a function of the fiber aspect ratio $\omega$, and part (b), $\bar{\lambda}_{\text {crit }}$ for fiber aspect ratios of 2,5 , and 10 , as a function of $\bar{\theta}$. An important observation that can be made from Fig. 11(a) is that, irrespectively of the loading angle, elastomers reinforced with fibers of higher aspect ratio are more unstable. Note also that unlike the $\bar{\lambda}_{\text {crit }}$ for $\bar{\theta}=0^{\circ}$ which exhibits a vertical asymptote at $\omega=1$, the $\bar{\lambda}_{\text {crit }}$ for misaligned loadings (i.e., $\bar{\theta} \neq 0^{\circ}$ ) reaches a maximum finite value at a certain $\omega>1$ beyond which no loss of ellipticity is detected. It is further noted from this figure that the homogenized material becomes unstable at larger stretches with increasing values of the loading angle. This point is more clearly illustrated by Fig. 11(b). In fact, Fig. 11(b) shows that beyond a certain threshold (depending on the aspect ratio $\omega$ ) in $\bar{\theta}$, the homogenized elastomer does not lose strong ellipticity. Physically, the results shown in Fig. 11 for rigidly reinforced, incompressible elastomers indicate that the compressive component of the applied loading along the longest in-plane axes of the fibers, together with the fiber aspect ratio, are the two major elements governing the macroscopic stability of the material. Indeed, the higher the aspect ratio and the higher the compressive deformation along the longest in-plane axes of the fibers, the more unstable the material is.

\subsection{Aligned pure shear loading: compliant fibers and compressible matrix}

Fig. 12 presents the effective behavior as predicted by the second-order method for a compressible, Neo-Hookean elastomer reinforced with stiffer Neo-Hookean fibers of initially elliptical cross section under aligned pure shear loading with $\bar{\theta}=0^{\circ}$. The fibers and the matrix are characterized by Neo-Hookean stored-energy functions such that $\mu^{(2)} / \mu^{(1)}=\kappa^{(2)} / \kappa^{(1)}=t$, with $t$ denoting the contrast. Results are shown for an initial fiber concentration of $c_{0}=30 \%$. Part (a) shows the effective stress $\bar{S}=\mathrm{d} \widehat{W} / \mathrm{d} \bar{\lambda}$ for a contrast of $t=10$ and initial fiber aspect ratios of $\omega_{0}=1,2,5$, and 10 , as a function of the
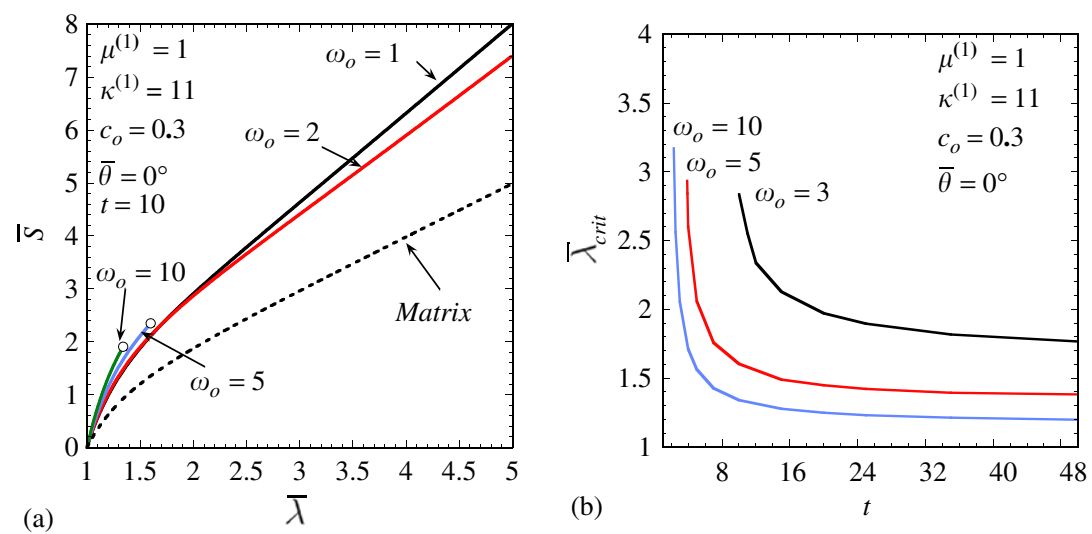

Fig. 12. Second-order estimates for the effective behavior of reinforced elastomers subjected to aligned pure shear loading $\left(\bar{\theta}=0^{\circ}\right)$. The results correspond to a compressible, Neo-Hookean matrix reinforced with an initial volume fraction of $30 \%$ of stiffer, Neo-Hookean fibers of initially elliptical cross section. (a) The effective stress $\bar{S}=$ $\mathrm{d} \widehat{W} / \mathrm{d} \bar{\lambda}$ for contrasts of $t=\mu^{(2)} / \mu^{(1)}=\kappa^{(2)} / \kappa^{(1)}=10$ and various aspect ratios, as a function of the applied stretch $\bar{\lambda}$. (b) The critical stretch $\bar{\lambda}_{\text {crit }}$ at which loss of strong ellipticity of the homogenized reinforced elastomer takes place for various fiber aspect ratios, as a function of the contrast $t$. 
macroscopic stretch $\bar{\lambda}$, and part (b), the critical stretch $\bar{\lambda}_{\text {crit }}$ at which the homogenized elastomer loses strong ellipticity for initial fiber aspect ratios of $\omega_{0}=3,5$, and 10 , as a function of the contrast $t$. First, note the parallel between Figs. 12(a) and 5(b). (Recall that the fibers were taken to be rigid in Fig. 5(b).) Observe that the results shown in Fig. 12(a) exhibit a significant reinforcement effect with the addition of stiffer fibers, but less pronounced than that one observed in Fig. 5(b). Also, unlike the results shown in Fig. 5(b), where higher values of the aspect ratio of the fibers consistently led to a stiffer overall behavior, higher aspect ratios of the fibers lead here to a stiffer overall behavior of the material only for small deformations, whereas the opposite is true for large stretches. Furthermore, unlike the results shown in Fig. 5(b), where loss of strong ellipticity of the homogenized elastomer was always detected at some finite stretch for any aspect ratio $\omega>1$, the results shown in Fig. 12(a) are found to lose ellipticity only for sufficiently large initial aspect ratios $\left(\omega_{0}=5\right.$ and 10$)$. These discrepancies will be shown to be connected with the evolution of the microstructure. In Fig. 12(b), it is seen that, under the given loading conditions, the type of reinforced elastomers considered here become more unstable with increasing stiffness of the fibers. In fact, for small values of $\omega$, loss of strong ellipticity is detected only for sufficiently large values of $t$. This figure also shows that, similar to the results for rigidly reinforced elastomers, higher initial fiber aspect ratios lead to a more unstable overall behavior. Finally, it should be remarked that the loss of strong ellipticity shown in Fig. 12(b) is due to the vanishing of the effective shear modulus $\widehat{\mathscr{L}}_{1212}^{c}$ in condition (42), corresponding, once again, to the possible "flopping" of the fibers.

Fig. 13 provides plots associated with the results shown in Fig. 12(a) for the evolution of the underlying microstructure in compressible, Neo-Hookean elastomers reinforced with stiffer Neo-Hookean fibers of contrast $t=10$. Results are shown for an initial fiber volume fraction of $c_{0}=30 \%$, and initial fiber aspect ratios of $\omega_{0}=1,2,5$, and 10 , as a function of the applied stretch $\bar{\lambda}$. Part (a) shows the evolution of the volume fraction of the fibers $c$, and (b), the evolution of the aspect ratio $\omega$. It is evident from Fig. 13(a) that the volume fraction of the fibers does not change significantly under aligned pure shear deformations,
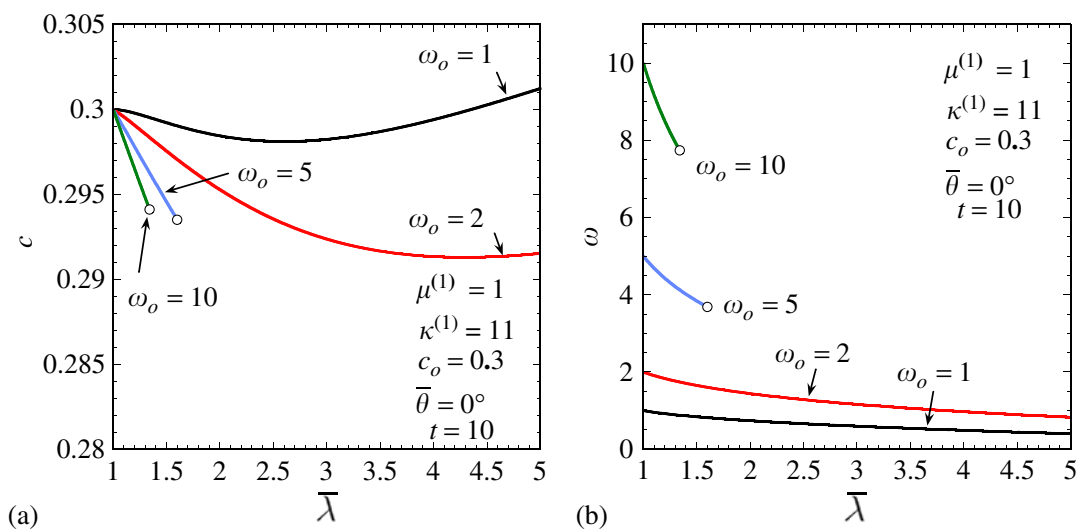

Fig. 13. Aligned pure shear loading $\left(\bar{\theta}=0^{\circ}\right)$ of a compressible, Neo-Hookean elastomer reinforced with stiffer Neo-Hookean fibers of initially elliptical cross section. Results are shown for a contrast of $t=\mu^{(2)} / \mu^{(1)}=\kappa^{(2)} / \kappa^{(1)}=10$, various initial fiber aspect ratios, and initial fiber concentration of $30 \%$, as a function of the applied stretch $\bar{\lambda}$. (a) The evolution of the volume fraction of the fibers. (b) The evolution of the aspect ratio of the fibers. 
irrespectively of the initial aspect ratio of the fibers. This result is due to the facts that the macroscopic deformation applied here is isochoric, and that both, the matrix and the fibers, were taken to be fairly incompressible (i.e., $\kappa^{(1)}=11$ and $\kappa^{(2)}=110$ ). More insightful are the results shown in Fig. 13(b), where it is seen that the aspect ratio of the fibers decreases significantly as a function of the macroscopic applied stretch $\bar{\lambda}$, as it might be expected on physical grounds since compression is being applied along the longest inplane axes of the fibers. It is also worthwhile to remark from this figure that aspect ratios with a higher initial value exhibit a higher rate of decrease as a function of $\bar{\lambda}$. Recall now that the amount of compressive loading along the longest in-plane axes of the fibers, together with the fiber aspect ratio, have been established to be the two major elements governing the macroscopic instabilities of the type of rigidly reinforced elastomers of interest in this work. This statement holds true more generally for elastomers reinforced with stiffer fibers of finite stiffness. In this regard, it is relevant to remark that for the cases shown in Fig. 13(b) the microstructure evolves into a more stable configuration, as the aspect ratio of the fibers decreases with increasing $\bar{\lambda}$. In particular, note that for the case of $\omega_{0}=2$, the current aspect ratio $\omega$ evolves into values smaller than unity for sufficiently large stretches. Once $\omega<1$, the longest axes of the fibers become aligned with the principal direction of tensile loading, a configuration for which the material remains strongly elliptic. This is in contrast to the results presented in Fig. 5(b) for rigid fibers with $\omega=2$, which were found to admit loss of strong ellipticity at finite stretch. Evidently, in this latter case, the aspect ratio of the rigid fibers remained fixed along the deformation path, thus constraining the microstructure from evolving into a more stable configuration. Note that for the cases of $\omega_{0}=5$ and $\omega_{0}=10$ in Fig. 13(b) loss of strong ellipticity takes place before the current aspect ratio reaches 1 .

\subsection{Simple shear loading: rigid fibers and incompressible matrix}

Fig. 14 presents second-order estimates for the effective behavior of an incompressible, Neo-Hookean elastomer reinforced with rigid fibers of elliptical cross section under simple
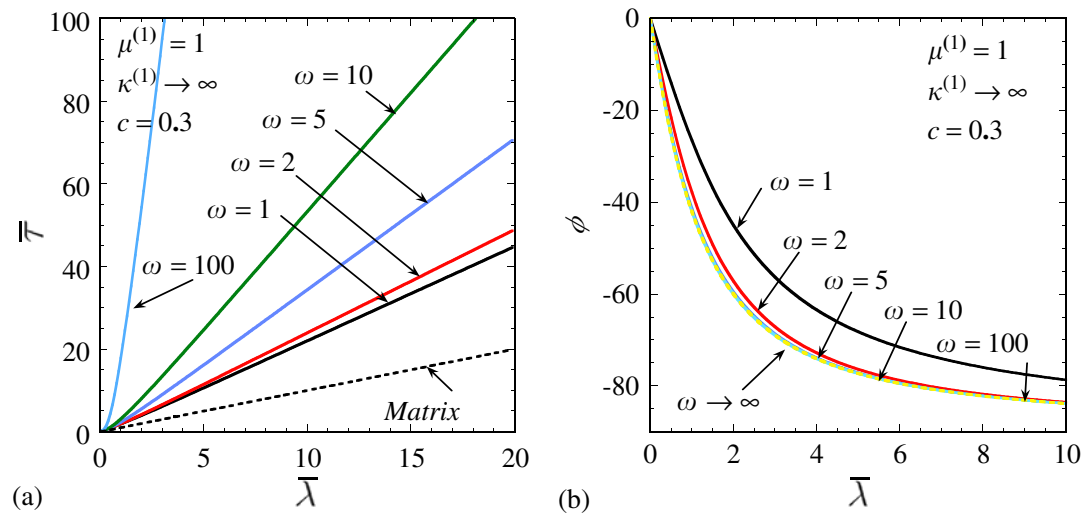

Fig. 14. Second-order estimates for the effective behavior of rigidly reinforced elastomers subjected to simple shear "perpendicular" to the fibers. The results correspond to an incompressible, Neo-Hookean, matrix phase with fiber concentration $c=30 \%$ and various values of the fiber aspect ratio $\omega$, and are shown as a function of the applied macroscopic shear $\bar{\gamma}$. (a) The effective stress $\bar{\tau}=\mathrm{d} \widehat{W} / \mathrm{d} \bar{\gamma}$. (b) The average angle of rotation of the fibers $\phi$. 
shear loading "perpendicular" to the fibers, i.e., $\overline{\mathbf{F}}=\mathbf{I}+\bar{\gamma} \mathbf{e}_{1} \otimes \mathbf{e}_{2}$. Results are shown for fiber aspect ratios of $1,2,5,10$, and 100, and fiber concentration of $30 \%$, as a function of the applied macroscopic shear $\bar{\gamma}$. Part (a) shows the effective stress $\bar{\tau}=\mathrm{d} \widehat{W} / \mathrm{d} \bar{\gamma}$, and (b), the average angle of rotation of the fibers $\phi$. Note that in this case the applied macroscopic rotation tensor is given by $\overline{\mathbf{R}}=1 / \sqrt{4+\bar{\gamma}^{2}}\left(2\left(\mathbf{e}_{1} \otimes \mathbf{e}_{1}+\mathbf{e}_{2} \otimes \mathbf{e}_{2}\right)+\bar{\gamma}\left(\mathbf{e}_{1} \otimes \mathbf{e}_{2}-\mathbf{e}_{2} \otimes \mathbf{e}_{1}\right)\right)$ so that $\bar{\psi}=-\arcsin \left(\bar{\gamma} / \sqrt{4+\bar{\gamma}^{2}}\right)$. It is observed from Fig. 14(a) that, similar to all previous cases, the addition of rigid fibers into the elastomeric matrix produces a significant reinforcement effect on the overall response of the material. Furthermore, this reinforcement effect becomes more pronounced for higher values of the fiber aspect ratio. This is consistent with the fact that in the limit $\omega \rightarrow \infty$ (as the microstructure tends to a simple laminate) the material becomes rigid under simple shear "perpendicular" to the fibers. Fig. 14(b) shows that all fibers, irrespectively of their aspect ratio, undergo a monotonic clockwise rotation (relative to the fixed frame of reference) with $\phi \rightarrow-90^{\circ}$ as $\bar{\gamma} \rightarrow \infty$, tending to align their longest in-plane axes with the principal tensile direction of the right stretch tensor $\overline{\mathbf{U}}$. As it was the case for pure shear at a fixed angle, fibers with higher aspect ratio rotate faster. In this regard, it is noted that the result for $\omega \rightarrow \infty$ has been included in Fig. 14(b) only for reference purposes. (Recall that the material behaves rigidly in this limit under the present loading conditions.) Finally, it is interesting to remark that no loss of strong ellipticity was found to take place in these materials under the given loading conditions.

Fig. 15 presents similar results for simple shear loading "parallel" to the fibers, i.e., $\overline{\mathbf{F}}=\mathbf{I}+\bar{\gamma} \mathbf{e}_{2} \otimes \mathbf{e}_{1}$. In this case, the applied macroscopic rotation tensor is given by $\overline{\mathbf{R}}=$ $1 / \sqrt{4+\bar{\gamma}^{2}}\left(2\left(\mathbf{e}_{1} \otimes \mathbf{e}_{1}+\mathbf{e}_{2} \otimes \mathbf{e}_{2}\right)+\bar{\gamma}\left(\mathbf{e}_{2} \otimes \mathbf{e}_{1}-\mathbf{e}_{1} \otimes \mathbf{e}_{2}\right)\right)$ so that $\bar{\psi}=\arcsin \left(\bar{\gamma} / \sqrt{4+\bar{\gamma}^{2}}\right)$. Similar to Fig. 14(a), the results presented in Fig. 15(a) show a reinforcement effect with the addition of rigid fibers. However, unlike Fig. 14(a), Fig. 15(a) shows that this reinforcement effect is more pronounced for lower values of the fiber aspect ratio. Indeed, it is seen that the reinforcement effect decreases monotonically with increasing aspect ratio, attaining the stiffest behavior at $\omega=1$, and the softest one at $\omega \rightarrow \infty$. (Recall that simple
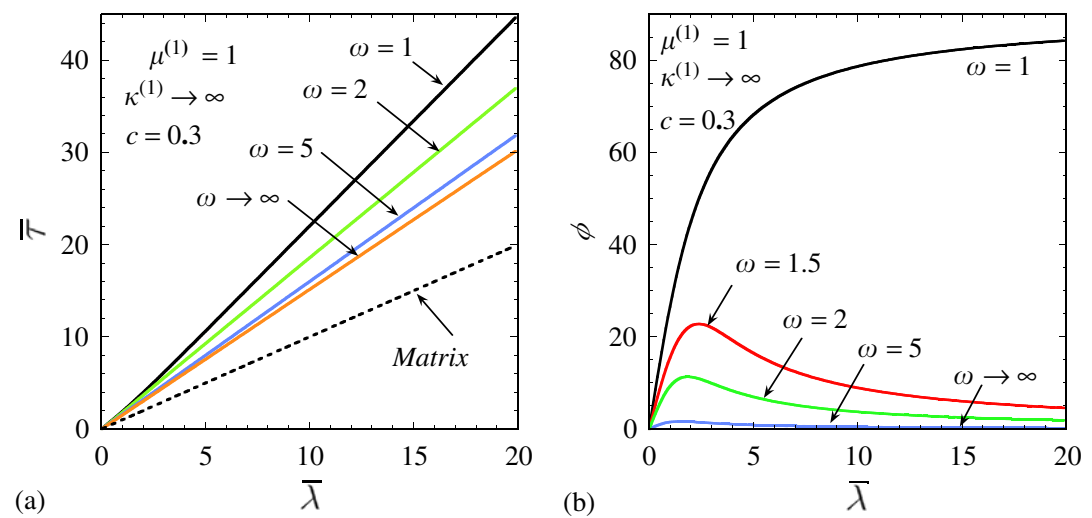

Fig. 15. Second-order estimates for the effective behavior of rigidly reinforced elastomers subjected to simple shear "parallel" to the fibers. The results correspond to an incompressible Neo-Hookean matrix phase with fiber concentration $c=30 \%$ and various values of the fiber aspect ratio $\omega$, and are shown as a function of the applied macroscopic shear $\bar{\gamma}$. (a) The effective stress $\bar{\tau}=\mathrm{d} \widehat{W} / \mathrm{d} \bar{\gamma}$. (b) The average angle of rotation of the fibers $\phi$. 
shear "parallel" to the fibers is the only admissible deformation for $\omega \rightarrow \infty$ in the context of rigid fibers.) Interestingly, this is exactly the same trend followed in the infinitesimal strain regime, where higher fiber aspect ratios lead to softer overall responses of the material. Fig. 15(b) shows that circular fibers rotate with the applied macroscopic rotation, that is, $\phi=\bar{\psi}$, and hence they undergo an anticlockwise monotonic rotation (relative to the fixed frame of reference) with $\phi \rightarrow 90^{\circ}$ as $\bar{\gamma} \rightarrow \infty$. On the other hand, elliptical fibers initially rotate anticlockwise, reaching a maximum value at certain shear $\bar{\gamma}$ after which they rotate clockwise asymptotically tending to $0^{\circ}$, thus aligning their longest in-plane axes with the principal tensile direction of the right stretch tensor $\overline{\mathbf{U}}$. Note that $\phi=0^{\circ}$ for all $\bar{\gamma}$ for the limiting case $\omega \rightarrow \infty$, in agreement with the exact result (for a laminate). Finally, akin to simple shearing "perpendicular" to the fibers, no loss of strong ellipticity was found to take place in these materials under the given conditions.

\section{Concluding remarks}

In this work, analytical estimates have been derived for the effective behavior, the microstructure evolution, and the onset of macroscopic instabilities in reinforced elastomers with random microstructures subjected to finite deformations, by making use of the framework developed in Part I of this paper. It is emphasized that this framework, which is built on an extension of the second-order homogenization method of Ponte Castañeda (2002), is in fact applicable to large classes of hyperelastic composites (see Lopez-Pamies and Ponte Castañeda, 2004b, for applications to porous elastomers).

A major result of this work is the strong influence of the evolution of the microstructure on the overall behavior of reinforced elastomers. Indeed, in the specific context of elastomers reinforced with rigid fibers (of elliptical cross section), the rotation (in the plane of the deformation) of the fibers when the composite is subjected to finite stretches has been identified as a potential softening mechanism. Physically, the fibers can "accommodate" some of the applied macroscopic deformation through rigid rotations, tending to align themselves with the tensile loading axis, which induces softer modes of deformation in the matrix phase. As a consequence, the overall response of the composite under loadings that promote large rotations of the underlying fibers tend to be much softer than those associated with loadings inducing smaller rotations (or no rotations at all).

Perhaps more significantly, the microstructure evolution not only has implications for the effective behavior, but also for the overall stability of the composite. In this regard, it has been shown that loss of strong ellipticity, corresponding to the possible development of shear-band type instabilities at a macroscopic length scale, can take place in strongly elliptic elastomers reinforced with stiff fibers (of elliptical cross section) at physically realistic levels of deformation. The underlying microscopic mechanism driving these macroscopic instabilities has been identified with the possible "flopping" of the fibers, due to a sufficiently large compressive component of the applied deformation along the long (in-plane) axes of the fibers. More specifically, in spite of the randomness, the assumed "elliptical symmetry" of the microstructure exhibits a preferred microstructural orientation. Thus, the "flopping" of the fibers provides a symmetry breaking mechanism leading to the possible development of a macroscopic instability. Indeed, in the limit as the fibers (and their two-point correlation function) are taken to be circular, so that the composite 
becomes isotropic, no loss of strong ellipticity is detected, as there are no symmetries to be broken.

Another important conclusion of this work is that the addition of rigid inclusions in an elastomer enhances the "lock-up" effect in the material due to the stretching of the polymer chains, provided that it is already present in the matrix phase. From the continuum point of view, this is consistent with the fact that, on average, the deformation in the matrix phase of a rigidly reinforced material is larger than the applied macroscopic deformation (since the rigid phase does not deform), leading then to a smaller overall lockup stretch. Physically, the effect of introducing rigid inclusions into an elastomer would make the polymeric chains wrap around the particles, which would be consistent with a smaller overall lock-up stretch upon deformation of the composite.

The encouraging results obtained in this work for two-dimensional microstructures provide ample motivation to carry out corresponding analyses for more general threedimensional, random microstructures, where comparisons with appropriate experimental data are feasible. Such analyses are in progress.

\section{Acknowledgements}

This work was supported by NSF Grant DMS-0204617.

\section{Appendix A. Incompressible limit for rigidly reinforced elastomers}

In this appendix, we sketch out the asymptotic analysis corresponding to the incompressible limit associated with the second-order estimate (10) for elastomers with isotropic matrix phases of form (16) reinforced with rigid fibers of volume fraction $c$ and aspect ratio $\omega$. As already discussed in the main body of text, only one of the two possible roots associated with the second-order estimates developed in this work provides physically sound results. Hence, only the limit associated with this root is presented here. It must be emphasized that the results generated by the following asymptotic analysis have been checked to be in agreement with the full numerical solution.

Based on numerical evidence from the results for finite $\kappa^{(1)}$, an expansion for the unknowns (i.e., $L_{1111}^{*}, L_{2222}^{*}, L_{1122}^{*}, L_{1212}^{*}$, and $\phi$ ) in this problem is attempted in the limit as $\kappa^{(1)} \rightarrow \infty$ of the following form:

$$
\begin{aligned}
& L_{1111}^{*}=a_{1} \Delta^{-1 / 3}+a_{2}+a_{3} \Delta^{1 / 3}+O\left(\Delta^{2 / 3}\right), \\
& L_{2222}^{*}=b_{1} \Delta^{-1 / 3}+b_{2}+b_{3} \Delta^{1 / 3}+O\left(\Delta^{2 / 3}\right), \\
& L_{1122}^{*}=d_{1} \Delta^{-1 / 3}+d_{2}+d_{3} \Delta^{1 / 3}+O\left(\Delta^{2 / 3}\right), \\
& L_{1212}^{*}=e_{2}+e_{3} \Delta^{1 / 3}+e_{4} \Delta^{2 / 3}+O(\Delta), \\
& \phi=\phi_{0}+\phi_{1} \Delta^{1 / 3}+O\left(\Delta^{2 / 3}\right),
\end{aligned}
$$

where $\Delta=1 / \kappa^{(1)}$ is a small parameter and $a_{1}, a_{2}, a_{3}, b_{1}, b_{2}, b_{3}, d_{1}, d_{2}, d_{3}, e_{2}, e_{3}, e_{4}, \phi_{0}$, and $\phi_{1}$ are unknown coefficients to be determined from the asymptotic analysis that follows. It will prove useful to spell out the corresponding expansions for the constrained components 
$L_{2121}^{*}$ and $L_{1221}^{*}$, as well as those for the components of $\mathbf{Y}=\overline{\mathbf{Q}}^{\mathrm{T}} \overline{\mathbf{R}}^{\mathrm{T}}\left(\hat{\mathbf{F}}^{(1)}-\overline{\mathbf{F}}\right) \overline{\mathbf{Q}}$, in the limit as $\kappa^{(1)} \rightarrow \infty$. Thus, introducing (46) in relations (21) for the components $L_{2121}^{*}$ and $L_{1221}^{*}$ can be shown to lead to the following expansions:

$$
\begin{aligned}
& L_{2121}^{*}=e_{2}+e_{3} \Delta^{1 / 3}+O\left(\Delta^{2 / 3}\right), \\
& L_{1221}^{*}=\left(\sqrt{a_{1} b_{1}}-d_{1}\right) \Delta^{-1 / 3}+\frac{a_{2} b_{1}+a_{1} b_{2}-\left(a_{1}+b_{1}\right) d_{2}}{2 \sqrt{a_{1} b_{1}}}-d_{2}+O\left(\Delta^{1 / 3}\right) .
\end{aligned}
$$

Similarly, introducing (46) in relations (24) for the components of $\mathbf{Y}$ leads to

$$
\begin{aligned}
& Y_{11}=x_{1}+x_{2} \Delta^{1 / 3}+x_{3} \Delta^{2 / 3}+x_{4} \Delta+O\left(\Delta^{4 / 3}\right), \\
& Y_{22}=y_{1}+y_{2} \Delta^{1 / 3}+y_{3} \Delta^{2 / 3}+y_{4} \Delta+O\left(\Delta^{4 / 3}\right), \\
& Y_{12} Y_{21}=p_{1}+p_{2} \Delta^{1 / 3}+p_{3} \Delta^{2 / 3}+p_{4} \Delta+O\left(\Delta^{4 / 3}\right), \\
& Y_{12}^{2}+Y_{21}^{2}=s_{1}+O\left(\Delta^{1 / 3}\right) .
\end{aligned}
$$

The explicit form of the coefficients in these expressions are too cumbersome to be included here. In any case, at this stage, it suffices to note that they are known in terms of the variables introduced in (46) (and higher-order correcting terms). In connection with relations (48), it is necessary to remark that the asymptotic expressions for the combinations $Y_{12} Y_{21}$ and $Y_{12}^{2}+Y_{21}^{2}$ have been specified, instead of those for the independent components $Y_{12}$ and $Y_{21}$, since, as discussed in the main body of the text, they are the relevant variables in this problem. For later use, it will also prove helpful to introduce the notation for the expansions of the constitutive function $g^{(1)}$ characterizing the matrix phase in the limit $\kappa^{(1)} \rightarrow \infty$ :

$$
\begin{aligned}
& g^{(1)}\left(\hat{I}^{(1)}\right)=\hat{g}=\hat{g}_{0}+\hat{g}_{1} \Delta^{1 / 3}+\hat{g}_{2} \Delta^{2 / 3}+O(\Delta), \\
& g_{I}^{(1)}\left(\hat{I}^{(1)}\right)=\hat{g}_{I}=\hat{g}_{0}^{\prime}+\hat{g}_{1}^{\prime} \Delta^{1 / 3}+\hat{g}_{2}^{\prime} \Delta^{2 / 3}+O(\Delta), \\
& g^{(1)}(\bar{I})=\bar{g}, \\
& g_{I}^{(1)}(\bar{I})=\bar{g}_{I},
\end{aligned}
$$

where $\hat{I}^{(1)}=\hat{\mathbf{F}}^{(1)} \cdot \hat{\mathbf{F}}^{(1)}, \hat{J}^{(1)}=\operatorname{det} \hat{\mathbf{F}}^{(1)}$, and appropriate smoothness has been assumed for $g^{(1)}$. Analogous expressions are defined for the material function $h^{(1)}$.

Now, by introducing expressions (46)-(49) in the generalized secant equation (7), as well as in Eq. (11) for the rigid rotation of the fibers, a hierarchical system of equations is obtained for the unknown coefficients in (46) and higher-order correcting terms. Thus, the equations of first order $O\left(\Delta^{-1}\right)$ can be shown to yield the following relations:

$$
\bar{J}=\bar{\lambda}_{1} \bar{\lambda}_{2}=1, \quad \bar{\lambda}_{2} x_{1}+\bar{\lambda}_{1} y_{1}+x_{1} y_{1}-p_{1}=0, \quad e_{2}=2 \hat{g}_{0}^{\prime} .
$$

Here, it is important to remark that condition $(50)_{1}$ is nothing more than the exact incompressibility constraint (26) specialized to plane-strain deformations. Moreover, it is noted that conditions (50) $)_{2}$ and (50) $)_{3}$ establish relations among the unknowns $a_{1}, a_{2}, b_{1}, b_{2}$, $d_{1}, d_{2}, e_{2}$, and $\phi_{0}$. Next, by making use of (50), the equations of second order $O\left(\Delta^{-2 / 3}\right)$ can 
be shown to ultimately render the conditions

$$
\bar{\lambda}_{2} x_{2}+\bar{\lambda}_{1} y_{2}+x_{1} y_{2}+x_{2} y_{1}-p_{2}=0, \quad e_{3}=2 \hat{g}_{1}^{\prime},
$$

which establish further relations among $a_{1}, a_{2}, a_{3}, b_{1}, b_{2}, b_{3}, d_{1}, d_{2}, d_{3}, e_{2}, e_{3}$, and $\phi_{0}$. Making use now of (50) and (51) in the equations of third order $O\left(\Delta^{-1 / 3}\right)$ gives

$$
\begin{aligned}
& b_{1}=\bar{\lambda}_{1}^{4} a_{1}, \\
& y_{1} \bar{\lambda}_{1}^{4} a_{1}+x_{1} \bar{\lambda}_{1}^{2} a_{1}+\bar{\lambda}_{1}\left(\bar{\lambda}_{1}^{2} a_{1}-d_{1}\right)=0, \\
& \bar{\lambda}_{2} x_{3}+\bar{\lambda}_{1} y_{3}+x_{1} y_{3}+y_{1} x_{3}+x_{2} y_{2}-p_{3}=d_{1}-\bar{\lambda}_{1}^{2} a_{1}, \\
& e_{4}=2 \hat{g}_{2}^{\prime}, \\
& \phi_{0}=\varphi+\bar{\psi},
\end{aligned}
$$

where

$$
\cos \varphi=\frac{(c-1) d_{1}}{c \bar{\lambda}_{1}\left(1+\bar{\lambda}_{1}^{2}\right) a_{1}}+\frac{(1+c) \bar{\lambda}_{1}}{c\left(1+\bar{\lambda}_{1}^{2}\right)},
$$

and $\bar{\psi}$ denotes the angle associated with the macroscopic rotation tensor $\overline{\mathbf{R}}$.

Finally, with the help of relations (50)-(53), the equations of fourth order $O(\Delta)$ can be shown to yield

$$
\begin{aligned}
& b_{2}-\bar{\lambda}_{1}^{4} a_{2}=\frac{2\left(1-\bar{\lambda}_{1}^{4}\right)}{d_{1}-\bar{\lambda}_{1}^{2} a_{1}}\left(d_{1} \hat{g}_{0}^{\prime}+a_{1}\left(\hat{g}_{0}^{\prime}-2 \bar{g}_{I}\right) \bar{\lambda}_{1}^{2}\right), \\
& 2 a_{1}^{2} \bar{\lambda}_{1}^{4}\left(a_{1} \bar{\lambda}_{1}^{2}-d_{1}\right)\left(\bar{\lambda}_{2} x_{2}+\bar{\lambda}_{1} y_{2}\right)-d_{1}^{2}\left(a_{2}-2 \hat{g}_{0}^{\prime}\right) \bar{\lambda}_{1}^{2} \\
& \quad-2 a_{1}^{2} \bar{\lambda}_{1}\left[d_{2} \bar{\lambda}_{1}^{3}+2\left(\hat{g}_{0}^{\prime}-\bar{g}_{I}\right)\left(\bar{\lambda}_{1}^{5}+x_{1}\left(\bar{\lambda}_{1}^{4}-1\right)\right)+\mu^{(1)} \bar{\lambda}_{1}^{3}\right] \\
& \quad+2 a_{1} d_{1}\left[\bar{g}_{I}^{\prime}\left(2-4 \bar{\lambda}_{1}^{4}\right)+2 \hat{g}_{0}^{\prime}\left(\bar{\lambda}_{1}^{4}-1\right)+\bar{\lambda}_{1}^{2}\left(d_{2}+a_{2} \bar{\lambda}_{1}^{2}+\mu^{(1)}\right)\right]=0, \\
& \bar{\lambda}_{2} x_{4}+\bar{\lambda}_{1} y_{4}+x_{1} y_{4}+y_{1} x_{4}+x_{2} y_{3}+x_{3} y_{2}-p_{4}=d_{2}+\mu^{(1)} \\
& \quad-2^{-1} \bar{\lambda}_{1}^{-2}\left(b_{2}+a_{2} \bar{\lambda}_{1}^{4}-2 \hat{g}_{0}^{\prime}\left(\bar{\lambda}_{1}^{4}+1\right)\right), \\
& e_{5}=2 \hat{g}_{3}^{\prime}, \\
& \cos \phi_{1}=G\left(a_{1}, d_{1}, a_{2}, d_{2}\right) \sin \phi_{0},
\end{aligned}
$$

where $G$ is (a known function of its arguments) too cumbersome to be included here, and use has been made of the facts that $\hat{h}_{o}=\bar{h}=0$ and $\hat{h}_{o}^{\prime}=\bar{h}_{J}=-\mu^{(1)}$.

Although the above system of algebraic, nonlinear equations is not closed (i.e., there are more unknowns than equations), it is possible to solve for the appropriate combinations of coefficients introduced in (46), which allows the determination of the leading order term of $\phi$, as well as the leading order term of the components of $\mathbf{Y}$ in the limit $\kappa^{(1)} \rightarrow \infty$. The results read as follows:

$$
\phi_{0}=\varphi+\bar{\psi}
$$




$$
\begin{aligned}
x_{1}= & \frac{c}{1-c}(\bar{\lambda}-\cos \varphi), \\
y_{1}= & \frac{c}{1-c}\left(\bar{\lambda}^{-1}-\cos \varphi\right), \\
p_{1}= & \frac{c}{\bar{\lambda}(1-c)^{2}}\left((2-c) \bar{\lambda}-\left(\bar{\lambda}^{2}+1\right) \cos \varphi+c \bar{\lambda} \cos ^{2} \varphi\right), \\
s_{1}= & \frac{c}{2 \omega(1-c)^{2} \bar{\lambda}^{2}}\left[-4(1+(\omega-1) \omega)\left(\bar{\lambda}+\bar{\lambda}^{3}\right) \cos \varphi\right. \\
& +2\left(\omega(c-4) \bar{\lambda}^{2}+\left(1+\bar{\lambda}^{2}\right)^{2}+\omega^{2}\left(1+\bar{\lambda}^{2}\right)^{2}-\omega c \bar{\lambda}^{2} \cos (2 \varphi)\right) \\
& \left.-2\left(\omega^{2}-1\right)\left(\bar{\lambda}^{4}-1\right) \cos (2 \bar{\theta}) \sin ^{2} \varphi+\left(\omega^{2}-1\right)\left(\bar{\lambda}^{4}-1\right) \sin (2 \bar{\theta}) \sin (2 \varphi)\right],
\end{aligned}
$$

respectively, where the angle $\varphi$ satisfies expression (30) given in the text. Here, $\bar{\lambda}=\bar{\lambda}_{1}=\bar{\lambda}_{2}^{-1}$, and $\bar{\theta}$ denotes the orientation of the in-plane principal axes of $\overline{\mathbf{U}}$ in agreement with the notation employed in Section 3.2.2.

Finally, it is straightforward to show that the leading order term of the expansion of the second-order estimate (10) in the limit of incompressibility may be ultimately written in terms of the leading order term of $\phi(55)$ and $\mathbf{Y}$ (56). The final explicit expression is given by (27) in the text.

\section{References}

Chadwick, P., Ogden, R., 1971. On the definition of elastic moduli. Arch. Ration. Mech. Anal. 44, 41-53.

Gent, A.N., 1996. A new constitutive relation for rubber. Rubber Chem. Technol. 69, 59-61.

Geymonat, G., Müller, S., Triantafyllidis, N., 1993. Homogenization of nonlinearly elastic materials, macroscopic bifurcation and macroscopic loss of rank-one convexity. Arch. Ration. Mech. Anal. 122, 231-290.

Hill, R., 1979. On the theory of plane strain in finitely deformed compressible materials. Math. Proc. Cambridge Philos. Soc. 86, 161-178.

Lopez-Pamies, O., Ponte Castañeda, P., 2004a. Second-order homogenization estimates incorporating field fluctuations in finite elasticity. Math. Mech. Solids 9, 243-270.

Lopez-Pamies, O., Ponte Castañeda, P., 2004b. Second-order estimates for the macroscopic response and loss of ellipticity in porous rubbers at large deformations. J. Elasticity 76, 247-287.

Lopez-Pamies, O., Ponte Castañeda, P., 2006. On the overall behavior, microstructure evolution, and macroscopic stability in reinforced rubbers at large deformations. I. Theory. J. Mech. Phys. Solids, in press, doi:10.1016/j.jmps.2005.10.006.

Ogden, R., 1978. Extremum principles in non-linear elasticity and their application to composites. I. Theory. Int. J. Solids Struct. 14, 265-282.

Ponte Castañeda, P., 1989. The overall constitutive behavior of nonlinearly elastic composites. Proc. R. Soc. London A 422, 147-171.

Ponte Castañeda, P., 2002. Second-order homogenization estimates for nonlinear composites incorporating field fluctuations. I. Theory. J. Mech. Phys. Solids 50, 737-757.

Ponte Castañeda, P., Tiberio, E., 2000. A second-order homogenization procedure in finite elasticity and applications to black-filled elastomers. J. Mech. Phys. Solids 48, 1389-1411.

Triantafyllidis, N.S., Nesterović, M.D., Schraad, M.W., 2005. Failure surfaces for finitely strained two-phase periodic solids under general in-plane loading. J. Appl. Mech., submitted for publication.

Willis, J.R., 1977. Bounds and self-consistent estimates for the overall moduli of anisotropic composites. J. Mech. Phys. Solids 25, 185-202.

Willis, J.R., 1981. Variational and related methods for the overall properties of composites. Adv. Appl. Mech. 21, $1-78$. 